\title{
First measurement of large area jet transverse momentum spectra in heavy-ion collisions
}

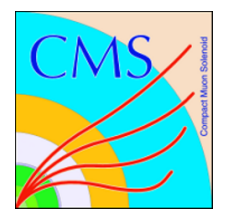

\section{The CMS collaboration}

E-mail: cms-publication-committee-chair@cern.ch

Abstract: Jet production in lead-lead $(\mathrm{PbPb})$ and proton-proton $(\mathrm{pp})$ collisions at a nucleon-nucleon center-of-mass energy of $5.02 \mathrm{TeV}$ is studied with the CMS detector at the LHC, using $\mathrm{PbPb}$ and pp data samples corresponding to integrated luminosities of $404 \mu \mathrm{b}^{-1}$ and $27.4 \mathrm{pb}^{-1}$, respectively. Jets with different areas are reconstructed using the anti- $k_{\mathrm{T}}$ algorithm by varying the distance parameter $R$. The measurements are performed using jets with transverse momenta $\left(p_{\mathrm{T}}\right)$ greater than $200 \mathrm{GeV}$ and in a pseudorapidity range of $|\eta|<2$. To reveal the medium modification of the jet spectra in $\mathrm{PbPb}$ collisions, the properly normalized ratio of spectra from $\mathrm{PbPb}$ and $\mathrm{pp}$ data is used to extract jet nuclear modification factors as functions of the $\mathrm{PbPb}$ collision centrality, $p_{\mathrm{T}}$ and, for the first time, as a function of $R$ up to 1.0. For the most central collisions, a strong suppression is observed for high- $p_{\mathrm{T}}$ jets reconstructed with all distance parameters, implying that a significant amount of jet energy is scattered to large angles. The dependence of jet suppression on $R$ is expected to be sensitive to both the jet energy loss mechanism and the medium response, and so the data are compared to several modern event generators and analytic calculations. The models considered do not fully reproduce the data.

KEywords: Hadron-Hadron scattering (experiments), Heavy-ion collision, Jets

ArXiv EPrint: 2102.13080 


\section{Contents}

1 Introduction 1

2 The CMS apparatus 3

3 Event selection 3

4 Monte Carlo simulations 4

5 Analysis method 4

5.1 Jet reconstruction and underlying event subtraction 4

5.2 Jet energy scale and resolution 6

5.3 Unfolding 8

6 Systematic uncertainties $\quad 8$

$\begin{array}{lll}7 & \text { Results } & 11\end{array}$

$\begin{array}{lll}8 & \text { Summary } & 19\end{array}$

$\begin{array}{lr}\text { The CMS collaboration } & 28\end{array}$

\section{Introduction}

Quantum Chromodynamics (QCD), the theory of the strong nuclear force, predicts that a deconfined state of quarks and gluons, called the quark-gluon plasma (QGP), should be produced at sufficiently high temperatures and densities [1]. In relativistic heavy ion collisions, the QGP is produced on an extremely short time scale [2,3]. A pair of partons (quarks or gluons) in the colliding nuclei can undergo a high transverse momentum $\left(p_{\mathrm{T}}\right)$ scattering, a process that occurs prior to the formation of the QGP. As the scattered partons pass through and interact with the QGP, they lose some of their energy, thereby acting as probes of the short-distance structure of the medium [4-8]. This parton energy loss, often referred to as "jet quenching", is related to the transport and thermodynamical properties of the QGP [9-12]. However, the details of the parton's interactions with the medium, as well as the relative importance of each interaction mechanism, are not yet fully understood [13-18].

A hard-scattered parton fragments and hadronizes into a collimated spray of particles. The fragmentation process coevolves with the QGP. The suppression of inclusive high- $p_{\mathrm{T}}$ hadrons in nucleus-nucleus collisions [19-26] provides evidence for jet quenching. Experimentally, final-state particles can be clustered into jets through the use of welldefined algorithms such as anti- $k_{\mathrm{T}}$ [27]. Various studies of jets and jet pairs, such as dijet 
$p_{\mathrm{T}}$ imbalance [28-30], modifications of the jet yield in the medium [31-37], electroweak boson-jet $p_{\mathrm{T}}$ imbalance [38, 39], jet fragmentation functions [40-42], missing $p_{\mathrm{T}}$ in dijet systems $[28,29,43]$, jet-track correlations [44], and the radial $p_{\mathrm{T}}$ profile of tracks within jets [45-49] have been studied. Complementary to these measurements, inclusive jet spectra reconstructed using different distance parameters $R$ in the anti- $k_{\mathrm{T}}$ algorithm are of great interest because they are less sensitive to hadronization effects than observables involving individual final-state hadrons. The value of $R$ defines the area of the reconstructed jet. By varying $R$, different fractions of energy from the quenched jet and the medium response will be included in the reconstructed jet. A differential study of the suppression versus $R$ provides new sensitivity to the QGP properties [50] and to the underlying jet quenching mechanism. In particular, theoretical models and generators based on perturbative QCD [50-52] and anti-de Sitter/conformal field theory correspondence [53] predict different dependences of the jet suppression on $R$.

Modifications to jet production can be quantified by the ratio of the inclusive jet yields per event in nucleus-nucleus (AA) collisions $\left(N^{\mathrm{AA}}\right)$ to those in proton-proton (pp) collisions $\left(N^{\mathrm{pp}}\right)$, scaled by the mean number of binary nucleon-nucleon $(\mathrm{NN})$ collisions $\left(\left\langle N_{\text {coll }}\right\rangle\right)[54]$. This ratio is called the nuclear modification factor $R_{\mathrm{AA}}$ and is defined as

$$
R_{\mathrm{AA}}\left(p_{\mathrm{T}}^{\mathrm{jet}}\right)=\frac{\mathrm{d} N^{\mathrm{AA}} / \mathrm{d} p_{\mathrm{T}}^{\text {jet }}}{\left\langle N_{\text {coll }}\right\rangle \mathrm{d} N^{\mathrm{pp}} / \mathrm{d} p_{\mathrm{T}}^{\text {jet }}}=\frac{\mathrm{d} N^{\mathrm{AA}} / \mathrm{d} p_{\mathrm{T}}^{\text {jet }}}{\left\langle T_{\mathrm{AA}}\right\rangle \mathrm{d} \sigma_{\text {inel }}^{\mathrm{pp}} / \mathrm{d} p_{\mathrm{T}}^{\text {jet }}},
$$

where $p_{\mathrm{T}}^{\text {jet }}$ is the transverse momentum of the jet. The $R_{\mathrm{AA}}$ is typically measured in bins of centrality, which characterizes the degree of overlap of the two colliding lead nuclei $[29,55]$. The nuclear overlap function $\left\langle T_{\mathrm{AA}}\right\rangle$ is defined as the ratio of $\left\langle N_{\text {coll }}\right\rangle$ to the total inelastic pp cross section, $\left\langle T_{\mathrm{AA}}\right\rangle=\left\langle N_{\text {coll }}\right\rangle / \sigma_{\text {inel }}^{\text {pp }}$, and can be calculated from a Glauber model of the nuclear collision geometry [55]. If the ratio is less than one, it indicates a transfer of energy out of the jet cone. Measurements of the dependence of jet spectra and nuclear modification factors on the jet distance parameter $R$ can help differentiate between competing models of parton energy loss mechanisms [56].

In studies of jet suppression from LHC Run 1 with lead-lead $(\mathrm{PbPb})$ collisions at a nucleon-nucleon center-of-mass energy of $\sqrt{s_{\mathrm{NN}}}=2.76 \mathrm{TeV}$, it was shown that the level of suppression is roughly independent of $p_{\mathrm{T}}^{\text {jet }}$ in the range $p_{\mathrm{T}}^{\text {jet }}=200-400 \mathrm{GeV}$ [33]. This suggests that the shape of the spectra is not significantly modified, and the modifications are predominantly through the overall number of jets. However, these initial measurements were statistically limited. At $\sqrt{s_{\mathrm{NN}}}=5.02 \mathrm{TeV}$, this measurement can be extended to higher $p_{\mathrm{T}}$. Furthermore, at this higher center-of-mass energy, partons traverse a medium of higher density and temperature.

In this paper, measurements of jet $R_{\mathrm{AA}}$ at $p_{\mathrm{T}}^{\text {jet }}>200 \mathrm{GeV}$ using $\mathrm{PbPb}$ collisions at $\sqrt{s_{\mathrm{NN}}}=5.02 \mathrm{TeV}$ are reported. The jets are reconstructed using the anti- $k_{\mathrm{T}}$ algorithm [27] with $R$ varying between 0.2 and 1.0. The results are presented as a function of $p_{\mathrm{T}}^{\text {jet }}$ in bins of $\mathrm{PbPb}$ event centrality. 


\section{The CMS apparatus}

The central feature of the CMS detector is a superconducting solenoid of $6 \mathrm{~m}$ internal diameter, providing a magnetic field of $3.8 \mathrm{~T}$. Within the solenoid volume are a silicon pixel and strip tracker, a lead tungstate crystal electromagnetic calorimeter (ECAL), and a brass and scintillator hadron calorimeter (HCAL), each composed of a barrel and two endcap sections. Hadron forward (HF) calorimeters extend the pseudorapidity coverage up to $|\eta|=5.2$ and are used for event selection. In the case of $\mathrm{PbPb}$ events, the HF signals are also used to determine the centrality class of the event. In the barrel section of the ECAL, an energy resolution of about $1 \%$ is achieved for unconverted or late-converting photons that have energies in the range of tens of $\mathrm{GeV}$. The remaining barrel photons have a resolution of about $1.3 \%$ up to $|\eta|=1$, rising to about $2.5 \%$ at $|\eta|=1.4$. In the endcaps, the resolution of unconverted or late-converting photons is about $2.5 \%$, while the remaining endcap photons have a resolution between 3 and 4\% [57]. When combining information from the entire detector, the jet energy resolution amounts typically to $15 \%$ at $10 \mathrm{GeV}, 8 \%$ at $100 \mathrm{GeV}$, and $4 \%$ at $1 \mathrm{TeV}$, to be compared to about 40,12 , and $5 \%$ obtained when the ECAL and HCAL calorimeters alone are used [58]. Muons are detected in gas-ionization chambers embedded in the steel flux-return yoke outside the solenoid. The silicon tracker measures charged particles within $|\eta|<2.5$. It consists of 1440 silicon pixel and 15148 silicon strip detector modules. For nonisolated particles of $1<p_{\mathrm{T}}<10 \mathrm{GeV}$ and $|\eta|<1.4$, the track resolutions are typically $1.5 \%$ in $p_{\mathrm{T}}$ and $25-90(45-150) \mu \mathrm{m}$ in the transverse (longitudinal) impact parameter [59]. Events of interest are selected using a twotiered trigger system [60]. The first level, composed of custom hardware processors, uses information from the calorimeters and muon detectors to select events at a rate of around $100 \mathrm{kHz}$ within a time interval of less than $4 \mu \mathrm{s}$. The second level, known as the high-level trigger, consists of a farm of processors running a version of the full event reconstruction software optimized for fast processing, and reduces the event rate to around $1 \mathrm{kHz}$ before data storage. A more detailed description of the CMS detector, together with a definition of the coordinate system used and the relevant kinematic variables, can be found in ref. [61].

\section{Event selection}

The event samples are recorded with dedicated jet triggers with different $p_{\mathrm{T}}^{\text {jet }}$ thresholds, the smallest of which is $p_{\mathrm{T}}^{\mathrm{jet}}>80 \mathrm{GeV}$ [39]. The efficiencies of the triggering algorithms are evaluated in data and are found to reach unity in both $\mathrm{pp}$ and $\mathrm{PbPb}$ collisions for jets considered in this paper $\left(p_{\mathrm{T}}^{\text {jet }}>200 \mathrm{GeV}\right)$. A number of requirements are made to the events to remove non-collision events (e.g., beam-gas interactions) and to select only inelastic hadronic collisions $[39,62]$. Both $\mathrm{pp}$ and $\mathrm{PbPb}$ events are required to have at least one reconstructed primary interaction vertex with a distance from the center of the nominal interaction region of less than $15 \mathrm{~cm}$ along the beam direction. In addition, in $\mathrm{PbPb}$ collisions the shapes of the clusters in the pixel detector have to be compatible with those produced by a genuine collision [63]. The $\mathrm{PbPb}$ collision events are also required to 


\begin{tabular}{|ccc|}
\hline Centrality & $\left\langle N_{\text {coll }}\right\rangle$ & $\left\langle T_{\mathrm{AA}}\right\rangle\left[\mathrm{mb}^{-1}\right]$ \\
\hline $0-10 \%$ & $1630_{-120}^{+120}$ & $23.2_{-0.7}^{+0.4}$ \\
$10-30 \%$ & $805_{-58}^{+55}$ & $11.5_{-0.4}^{+0.3}$ \\
$30-50 \%$ & $267_{-20}^{+20}$ & $3.82_{-0.21}^{+0.21}$ \\
$50-90 \%$ & $30.8_{-2.4}^{+3.5}$ & $0.440_{-0.032}^{+0.049}$ \\
\hline
\end{tabular}

Table 1. The values of $\left\langle N_{\text {coll }}\right\rangle$ and $\left\langle T_{\mathrm{AA}}\right\rangle$, and their uncertainties in $\sqrt{s_{\mathrm{NN}}}=5.02 \mathrm{TeV} \mathrm{PbPb}$ collisions for the centrality ranges used in this analysis [54].

have at least three towers in each of the HF detectors with energy deposits of more than $3 \mathrm{GeV}$ per tower. These criteria select $99 \%$ of inelastic hadronic $\mathrm{PbPb}$ collisions [29].

The collision centrality for $\mathrm{PbPb}$ events is determined using the total sum of transverse energy from the calorimeter towers in the HF region. The transverse energy distribution is used to divide the event sample into bins of percentage of the total hadronic interaction cross section [29]. The results in this paper are presented in four centrality intervals, where $0 \%$ corresponds to a full overlap of the two nuclei: $0-10,10-30,30-50$, and $50-90 \%$. The corresponding $\left\langle T_{\mathrm{AA}}\right\rangle$ and $\left\langle N_{\text {coll }}\right\rangle$ values used in this paper for the centrality intervals are listed in table 1.

\section{Monte Carlo simulations}

Several Monte Carlo (MC) simulated jet event samples are used to evaluate background components, efficiencies, misreconstructed jet rates (arising from upward fluctuations of the underlying event (UE) without a corresponding hard parton), jet energy corrections and jet energy resolutions (JER). Proton-proton collisions are generated with PYTHIA 8.212 [64], with the UE tune CUETP8M1 [65], as well as with PYTHIA6 [66], with the UE tune Z2 [67] with PDF set CTEQ6L1 [68]. For the $\mathrm{PbPb} \mathrm{MC}$ samples, each PYTHIA signal event is embedded into a $\mathrm{PbPb}$ collision event generated with HYDJET v1.8 [69], which is tuned to reproduce global event properties such as the charged-hadron $p_{\mathrm{T}}$ spectrum and particle multiplicity. The detailed simulation of the CMS detector response is performed using the GEANT4 package [70].

\section{Analysis method}

\subsection{Jet reconstruction and underlying event subtraction}

Particle candidates are reconstructed with the particle-flow (PF) algorithm [58], where information from different parts of the detector are combined to form an optimized description of the event. Jets are clustered from the PF candidates using the anti- $k_{\mathrm{T}}$ algorithm with distance parameters of $R=0.2,0.3,0.4,0.6,0.8$, and 1.0, as implemented in the FASTJET framework $[27,71]$. 
One of the main challenges to reconstructing jets in heavy-ion collisions is the additional soft UE coming from the QGP. In order to subtract the soft UE in $\mathrm{PbPb}$ collisions on an event-by-event basis, an iterative algorithm [72] is employed. The mean value, $\left\langle E_{\mathrm{PF}}\right\rangle$, and dispersion, $\sigma\left(E_{\mathrm{PF}}\right)$, of the transverse energies from the $\mathrm{PF}$ candidates are calculated in a number of $\eta$ bins $[29,38,73]$ for each event. Then, a two-step procedure is employed to account for the azimuthal modulation of background activity arising from the bulk properties of the QGP. In the first step, the so-called event plane angles $\left(\Phi_{\mathrm{EP}, 2}, \Phi_{\mathrm{EP}, 3}\right)$ for the second- and third-order harmonics of the azimuthal distribution are derived from the HF calorimeters $(3<|\eta|<5)$ [74]. This method of estimating the UE gives underlying energy estimations that are consistent with a previous analysis of photon- and Z-tagged jets in which event plane mixing was used [75]. The event plane angles are not corrected for detector effects since the only goal of this procedure is to obtain a better description of the modulation of the background level. For the second step, a fit to the azimuthal angle $(\phi$, in radians) distribution of charged-hadron $\mathrm{PF}$ candidates with $0.3<p_{\mathrm{T}}<3.0 \mathrm{GeV}$ and $|\eta|<1$ is performed. No explicit exclusion of regions close to the jet is performed, since their effect on the extracted parameters is negligible. The functional form of the fit is as follows:

$$
N(\phi)=N_{0}\left(1+2 v_{2} \cos \left(2\left[\phi-\Phi_{\mathrm{EP}, 2}\right]\right)+2 v_{3} \cos \left(3\left[\phi-\Phi_{\mathrm{EP}, 3}\right]\right)\right),
$$

where $N_{0}$ is the magnitude of average UE activity. The parameters $v_{2}$ and $v_{3}$ quantify the strengths of the collective behaviors of the UE known as "elliptic" and "triangular" flow, respectively. The event plane angles $\Phi_{\mathrm{EP}, 2}$ and $\Phi_{\mathrm{EP}, 3}$ are fixed to the result from the first step. A fit is performed per event to extract the parameters $N_{0}, v_{2}$, and $v_{3}$. The fit is discarded if the minimum required number of candidates (at least 10 entries in each bin) are not met, or if the reduced $\chi^{2}$ of the fit is greater than 2 . In this case, the background energy density is estimated as a flat distribution in $\phi$, without flow modulations.

An example of this procedure is shown for data in figure 1. The left plot shows the fit in the extraction region, along with a breakdown of the components of the fit. The right plot takes parameters extracted from mid-rapidity $(|\eta|<1)$ and renormalizes the function to data at forward-rapidity $(1<|\eta|<2)$. A good agreement of the shape for background modulations in the two $\eta$ ranges is observed.

Finally, the UE subtraction in $\mathrm{PbPb}$ collisions is performed using a constituent subtraction method [76]. This is a particle-by-particle approach that corrects the energy of each jet constituent based on the local average UE density $\rho(\eta, \phi)$. This density is assumed to factorize in $\eta$ and $\phi$ according to the form

$$
\rho(\eta, \phi)=\rho(\eta)\left(1+2 v_{2} \cos \left(2\left[\phi-\Phi_{\mathrm{EP}, 2}\right]\right)+2 v_{3} \cos \left(3\left[\phi-\Phi_{\mathrm{EP}, 3}\right]\right)\right) .
$$

Here $\rho(\eta)$ encodes the variation of the UE density as a function of $\eta$, and the flow parameters are determined in the previous fit. The average UE density $\rho(\eta)$ is calculated as the average energy in given $\eta$ bins, excluding regions overlapping with jets. In pp collisions, where the UE level is negligible, jets are reconstructed without UE subtraction. 


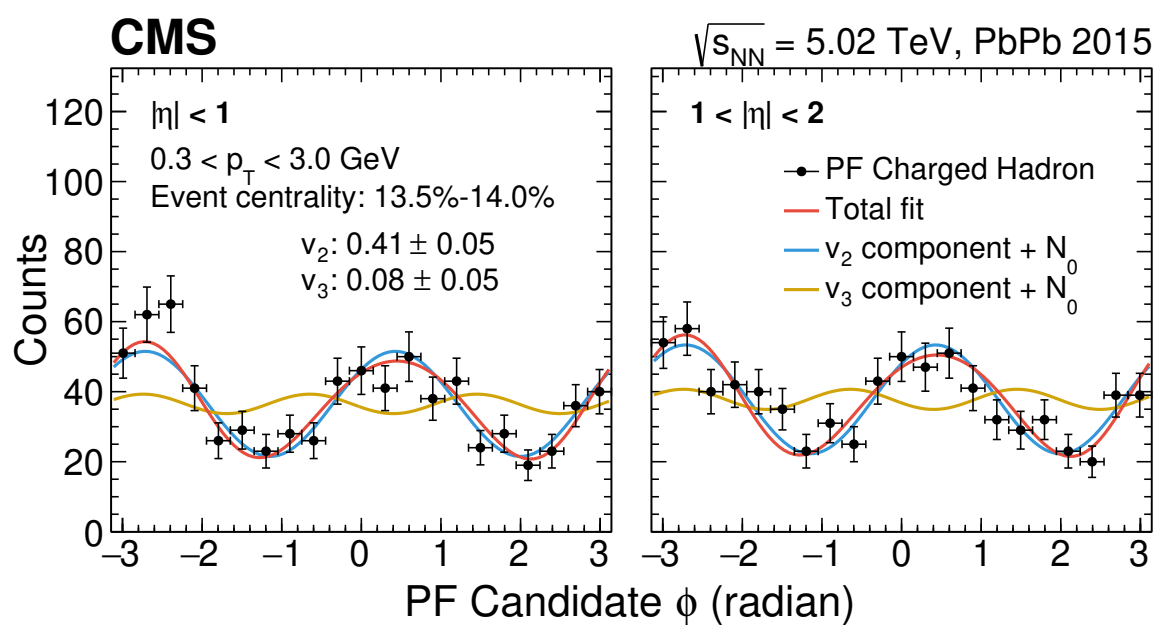

Figure 1. (Color online) Azimuthal angle distributions for a single $\mathrm{PbPb}$ event: $\phi$ modulations at mid-rapidity $|\eta|<1$ (left) and forward rapidity $1<|\eta|<2$ (right) of charged-hadron PF candidates. The $v_{2}$ (blue curve) and $v_{3}$ (yellow curve) of the flow components are shown, together with the total modulation used in the analysis to account for the background (red curve). The flow coefficients are extracted from the left plot and overlaid in the right plot.

\subsection{Jet energy scale and resolution}

Jet energy corrections are derived from simulation separately for $\mathrm{pp}$ and $\mathrm{PbPb}$ data following methods outlined in ref. [77]. The energy scales are verified with an energy balance method applied to dijet and photon+jet events in pp data. For this study, jets with $\left|\eta^{\text {jet }}\right|<2$ and (corrected) $p_{\mathrm{T}}^{\text {jet }}>160 \mathrm{GeV}$ are selected.

The $p_{\mathrm{T}}^{\text {jet }}$ binning of the analysis is chosen based on the jet energy resolution (JER) for each cone size and centrality. For pp events, the JER varies by less than $10 \%$ for different values of $R$. These variations reflect how the probability for energy to move into or out of the jet cone changes with cone sizes. Figure 2 shows the $\mathrm{PbPb}$ jet energy scale (JES, upper), defined as the reconstructed $p_{\mathrm{T}}^{\text {jet }}$ divided by the generated $p_{\mathrm{T}}^{\text {jet }}$, and JER (lower), for $R=0.2$ (left) and $R=1.0$ (right) as functions of the generated $p_{\mathrm{T}}^{\text {jet }}$. The JES is rather flat vs. $p_{\mathrm{T}}^{\text {jet }}$ while JER decreases with $p_{\mathrm{T}}^{\text {jet }}$. As expected, the resolution is worse for more central events and for larger values of $R$, because of the larger UE contribution that must be subtracted. For $R \leq 0.4$ the difference found in both pp and $\mathrm{PbPb}$ simulations between the JES of generated and reconstructed $p_{\mathrm{T}}^{\text {jet }}$ is below $2 \%$ at mid-rapidity $(|\eta|<1)$ and of order $4 \%$ for $(1<|\eta|<2)$.

A small nonclosure of up to $2 \%$ was observed for all values of $R$ in the peripheral $70-100 \% \mathrm{PbPb}$ bin, where the nonclosure is defined as the deviation of the corrected JES from unity. The UE in this bin is most comparable to that in pp collisions, and it is used to evaluate the performance of the jet algorithm with heavy ion reconstruction and subtraction in the absence of UE. This is necessary, as the difference in tracking and the subtraction of an $\mathrm{UE}$ in $\mathrm{PbPb}$, compared to $\mathrm{pp}$, results in modest performance changes even without a significant UE contribution. 


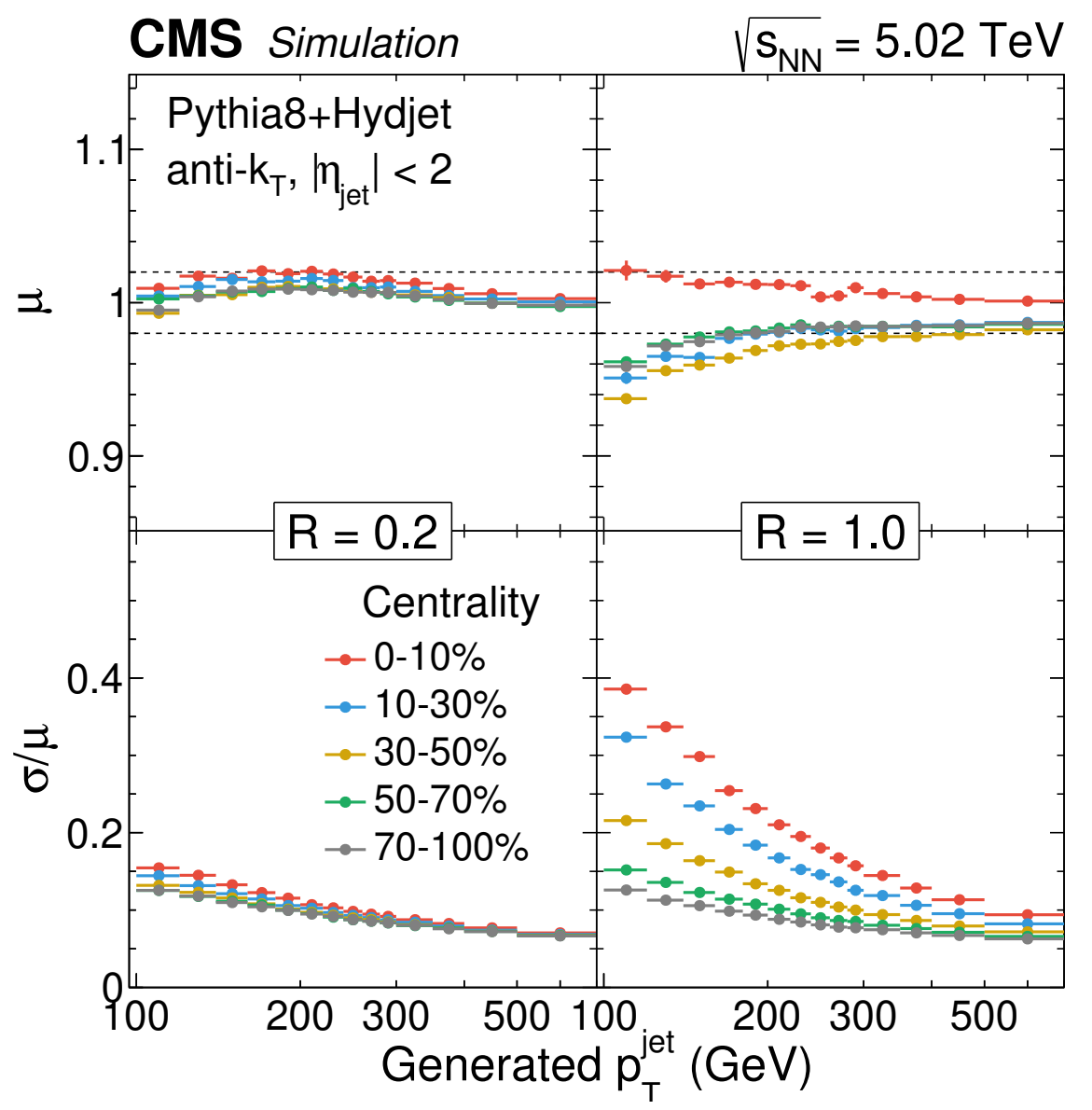

Figure 2. The energy scale $\mu$ (upper) and resolution $\sigma / \mu$ (lower) for $\mathrm{PbPb}$ anti- $k_{\mathrm{T}}$ jets with $\left|\eta^{\text {jet }}\right|<2$, as functions of generated $p_{\mathrm{T}}^{\text {jet }}$. The left (right) column shows jets with $R=0.2(1.0)$. Several different centrality classes are shown.

The $\phi$-modulation of $\rho$ shown in eq. (5.2) improves the jet resolution without introducing any biases to the energy scale. However, as can be seen in figure 2, there is evidence of over-subtraction for the largest values of $R$ at low $p_{\mathrm{T}}^{\text {jet }}$. This is because of uncertainties in the estimation of $\rho$. Errors in the estimation of $\rho$ tend to be handled much better for small $R$, as the subtraction scales with the multiplicative area. This over-subtraction causes the nonclosure to reach up to $4 \%$ for $R=1.0$ jets, as seen in figure 2. For smaller values of $R$ the nonclosure is below $2 \%$.

Another source of over-subtraction is caused by the flow-modulated subtraction. The minimum candidate count requirement for a good UE shape estimation does not account for the fact that jets could bias the fit. The over-subtraction occurs when a jet biases the flow modulation fit. While the fitting for $\phi$ modulation is turned off for events with a small number of tracks, events close to this threshold could still be affected by these biases, resulting in a source of nonclosure. 
Finally, for the most central events, where $\rho(\eta, \phi)$ ranges from $200-300 \mathrm{GeV}$ per unit area and the fluctuations are the largest, there is a global underestimation of the true UE, particularly in the forward region $(|\eta|>1)$. This occurs because towers within jets are nominally excluded in the estimation of $\rho$ to avoid bias from the hard process. In the most central events, upward fluctuations of the UE may cause some towers to be included in the jet and excluded from the UE. If too many towers are excluded, $\rho$ is underestimated. This underestimation of $\rho$ results in the largest uncertainty in the final $R_{\mathrm{AA}}$ and spectra for the most central bins. It is mitigated by setting an upper limit on the number of excluded towers, with a cutoff that is tuned to achieve the best performance.

\subsection{Unfolding}

Raw spectra are unfolded according to response matrices constructed using PYTHIA+HYDJET $\mathrm{MC}$ for $\mathrm{PbPb}$ and pure PYTHIA for pp results, in matched bins of $p_{\mathrm{T}}^{\text {jet }}, \eta^{\text {jet }}$, and for $\mathrm{PbPb}$ only, event centrality. The matrices are constructed with an $N_{\text {coll }}$ distribution that matches the expectations from data. The unfolding is done with the d'Agostini iteration with early stopping [78] as implemented in the RooUnFold package [79]. Examples of response matrices are shown in figure 3 for pp and $0-10 \% \mathrm{PbPb}$ collisions with $R=0.2$ and 1.0. Underflow bins are shown to account for bin migration. As expected, the matrices are more tightly diagonal for pp events than for $\mathrm{PbPb}$, and for $R=0.2$ than for $R=1.0$. The unfolded $\mathrm{pp}$ and $\mathrm{PbPb}$ spectra are then used to construct the $R_{\mathrm{AA}}$ distribution.

\section{Systematic uncertainties}

The systematic uncertainties in the spectra are estimated by varying analysis parameters one at a time within a reasonable range, propagating the change through the full analysis chain, and then considering the deviation from the nominal results. For $R_{\mathrm{AA}}$, any correlation between the uncertainties in the $\mathrm{pp}$ and $\mathrm{PbPb}$ spectra is accounted for by simultaneously changing the same parameter in the $\mathrm{pp}$ and $\mathrm{PbPb}$ analyses, calculating a new $R_{\mathrm{AA}}$ and taking the difference from the nominal result. This procedure produces a significant reduction in the uncertainty from data-simulation differences that impact JES and JER since the pp and $\mathrm{PbPb}$ were taken in run periods separated by just a few days. For ratios of $R_{\mathrm{AA}}$ between different jet radii, the luminosity and the $\left\langle T_{\mathrm{AA}}\right\rangle$ uncertainties cancel.

Finally, in the $R_{\mathrm{AA}}$ ratio between different radii, and the pp ratios of spectra between radii, there are statistical cancellations as the same jet may contribute to multiple $R$ spectra. These are accounted for by comparing ratios of spectra in pseudo-experiments generated independently from the spectra and those generated with the correlation between different $R$ taken from the data.

Figure 4 shows the principal systematic uncertainties as a function of $p_{\mathrm{T}}^{\text {jet }}$ for $R=$ 0.2 and 1.0, and for $\mathrm{pp}$ and $\mathrm{PbPb}$ collisions as a function of centrality. The dominant uncertainty arises from the JES. This tends to increase with $p_{\mathrm{T}}^{\text {jet }}$ and centrality but does not have a strong dependence upon $R$. The unfolding and JER uncertainties tend to 


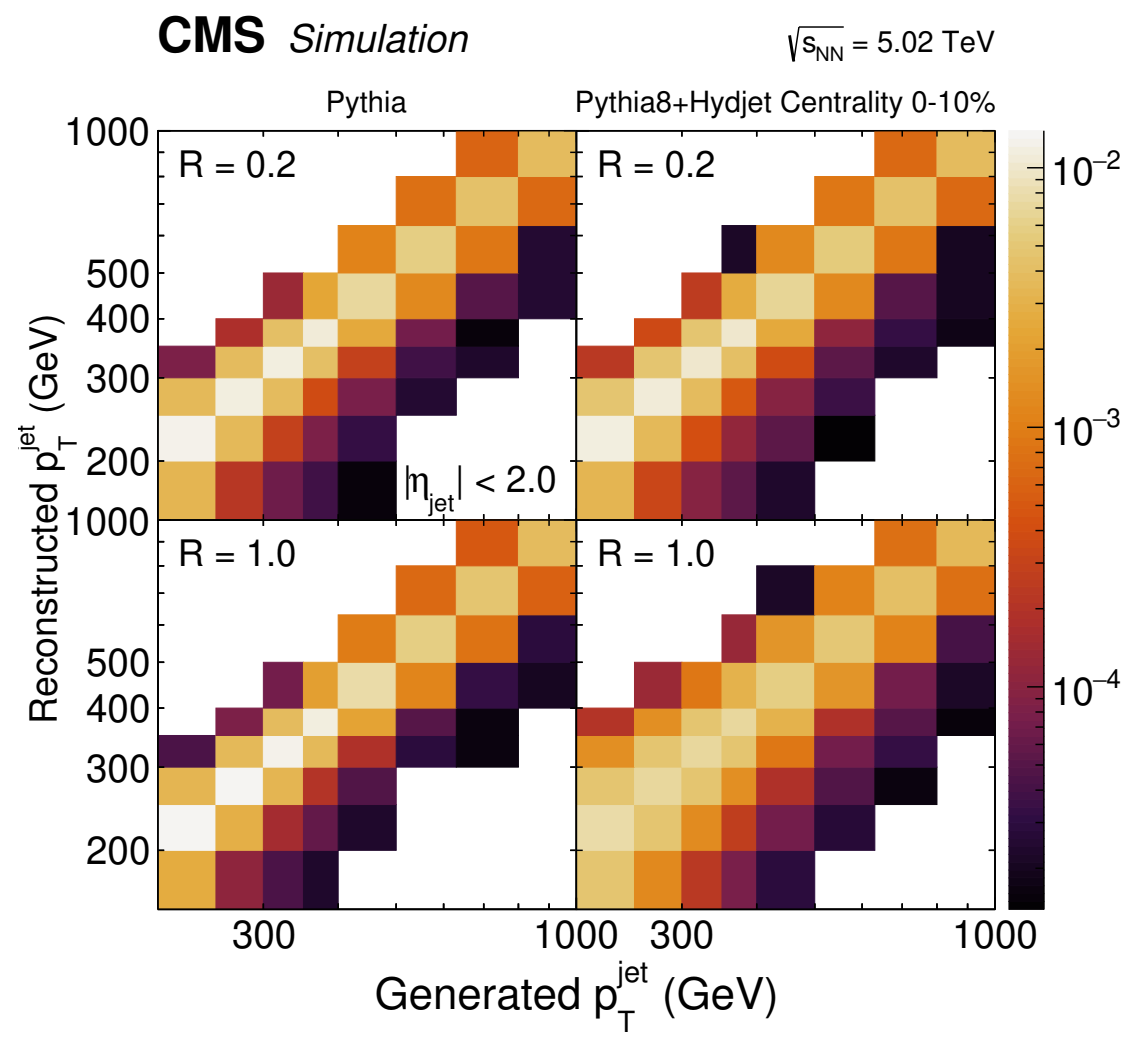

Figure 3. Response matrices in PYTHIA (left) and PYTHIA+HYDJET $0-10 \% \mathrm{PbPb}$ (right) events for jets reconstructed with $R=0.2$ (upper), $R=1.0$ (lower) and $\left|\eta^{\text {jet }}\right|<2$. The integral for each generated $p_{\mathrm{T}}^{\text {jet }}$ bin is normalized to unity.

decrease with $p_{\mathrm{T}}^{\text {jet }}$ and increase with centrality and $R$. The $\left\langle T_{\mathrm{AA}}\right\rangle$ uncertainty decreases from peripheral to central events and is independent of $p_{\mathrm{T}}^{\mathrm{jet}}$ and $R$.

The origins of these uncertainties are listed below in order of importance for $R_{\mathrm{AA}}$.

1. Jet energy scale. The uncertainty ranges from 15 to $20 \%$ and is dominated by the data-simulation difference. It consists of several components, summed in quadrature:

(a) Nonclosure in simulation. This uncertainty is evaluated as a function of centrality and $\eta$ but independently of $p_{\mathrm{T}}^{\text {jet }}$. It is estimated by varying data by the observed nonclosure in simulation, see figure 2, and then propagating this change through the analysis chain. In pp and peripheral (50-90\%) $\mathrm{PbPb}$ collisions, a $1 \%$ variation is made for all $\eta$. For $10-50 \%$ centrality, the variation is $1 \%$ within $\left|\eta^{\text {jet }}\right|<1$ and $2 \%$ for $1<\left|\eta^{\text {jet }}\right|<2$. For the most central $(0-10 \%)$ events, a $2 \%$ variation is made for jets within $\left|\eta^{\text {jet }}\right|<1$ and a $4 \%$ variation for $1<\left|\eta^{\text {jet }}\right|<2$.

(b) Data-simulation differences. A flat $2 \%$ variation is performed in all bins following the procedure used for the nonclosure uncertainties above. This uncertainty is dominant in the pp spectra, and comparable to the nonclosure uncertainty in semicentral and semiperipheral $\mathrm{PbPb}$ bins. 


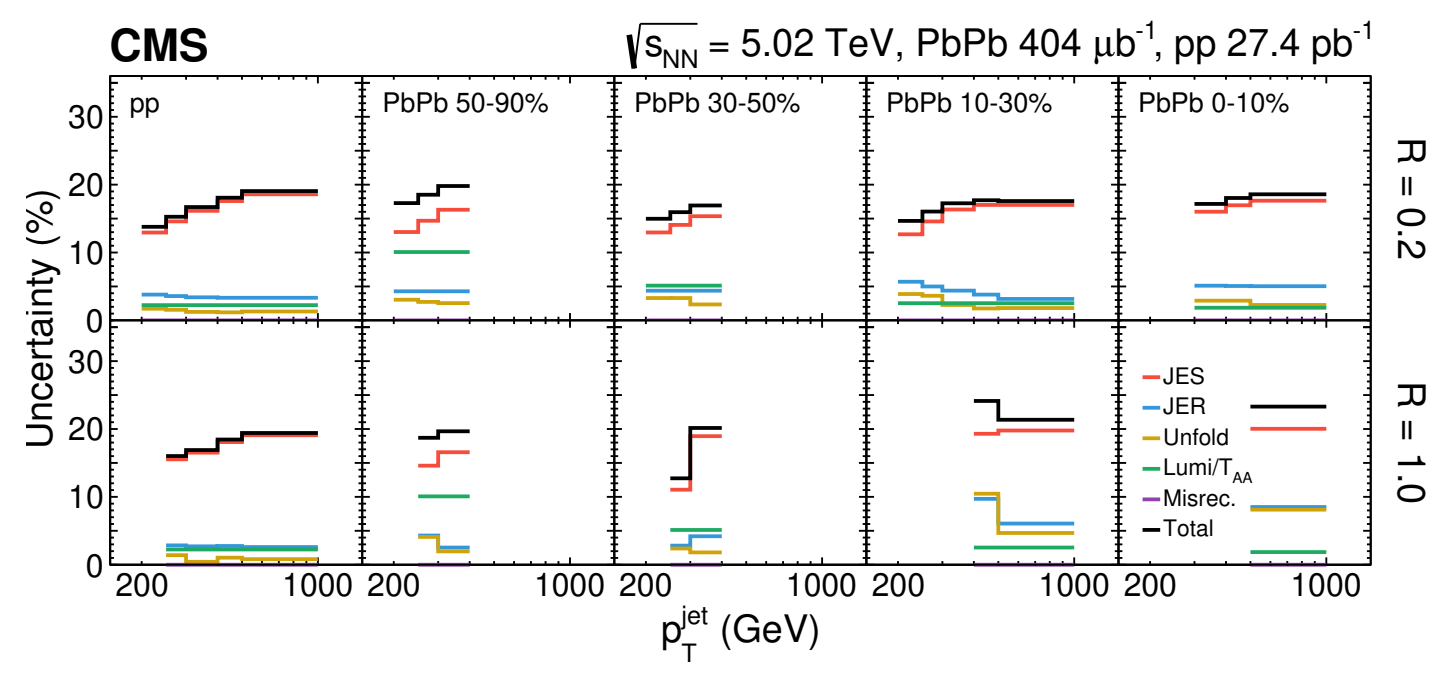

Figure 4. Relative systematic uncertainties for the spectra of anti- $k_{\mathrm{T}}$ jets within $\left|\eta^{\text {jet }}\right|<2.0$ for pp collisions (left), and $\mathrm{PbPb}$ with centrality classes 50-90\%, 30-50\%, 10-30\%, and 0-10\% (rightmost plot). The upper plots are for jets with $R=0.2$ and the lower plots for jets with $R=1.0$.

(c) Differences from the UE description between data and simulation. These differences are extracted by comparing random cone mean/widths between data and simulation, and the full difference is taken as a systematic uncertainty. As this is the centrality-dependent component of the JES, it does not cancel in the ratios between $\mathrm{pp}$ and $\mathrm{PbPb}$ data, and only cancels partially in $R$-dependent ratios of $R_{\mathrm{AA}}$.

2. Jet energy resolution.

(a) The JER uncertainty is extracted from simulation. This is subdominant compared to the data-simulation differences for spectra, but does not cancel in $R_{\mathrm{AA}}$.

(b) Jet energy resolution from data-simulation differences. The resolution in data is found to be 10 to $15 \%$ worse than that in simulation. To propagate this uncertainty, the simulation is first smeared by $10 \%$, such that central values are closer to those in data. The systematic uncertainty is estimated by applying an additional smearing on top of these new central values such that the resolution is increased by $10 \%$ in all bins. The effect is subdominant in part because the $p_{\mathrm{T}}^{\text {jet }}$ binning was chosen to minimize bin migration. Furthermore, there is partial cancellation in $R_{\mathrm{AA}}$, coming from the constant and stochastic terms of the resolution, which are partially shared between the $\mathrm{pp}$ and $\mathrm{PbPb}$ data.

3. Unfolding. This source of uncertainty is typically of order $5 \%$ with a maximum of 10\%. There are several components within this category:

(a) The choice of the prior. A variation of the nominal prior for the underlying $p_{\mathrm{T}}^{\mathrm{jet}}$ spectrum is done and propagated through the full analysis chain, including the response matrix. 


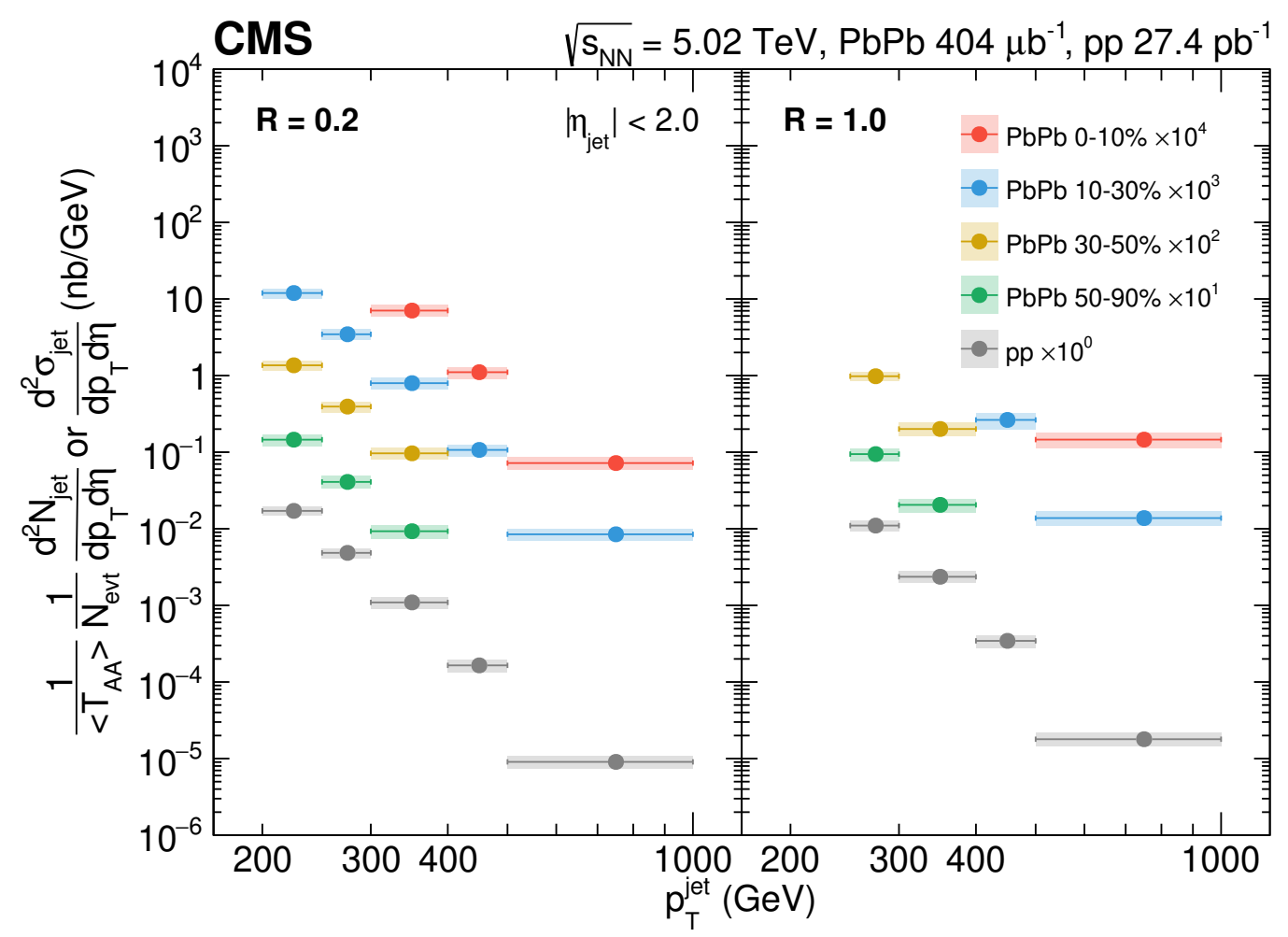

Figure 5. Spectra of jets with $\left|\eta^{\text {jet }}\right|<2.0$ for $R=0.2$ (left) and $R=1.0$ (right), for pp collisions and different centrality classes of $\mathrm{PbPb}$ collisions. The spectra are multiplied by successive factors of 10 for clarity. The statistical uncertainties are smaller than the marker sizes, while the systematic uncertainties are shown as shaded boxes. The markers are placed at the bin centers.

(b) Unfolding algorithm. The result is cross-checked with singular value decomposition unfolding [80].

4. Integrated luminosity and $\left\langle T_{\mathrm{AA}}\right\rangle$. The uncertainty in the integrated luminosity for pp collisions is $2.3 \%$ [81]. For the $\left\langle T_{\mathrm{AA}}\right\rangle$, the relative uncertainties vary between $3 \%$ for the $0-10 \%$ bin, to $11 \%$ in the most peripheral $50-90 \%$ bin [54]. The absolute uncertainties for each of the four values are listed in table 1.

5. Misreconstructed jets which arise from fluctuations of the UE. The contamination from these jets is evaluated from simulation, and it is found to be negligible in the considered kinematic range.

\section{Results}

The unfolded jet spectra as functions of $p_{\mathrm{T}}^{\text {jet }}$ for $R=0.2$ and 1.0 for both $\mathrm{pp}$ and $\mathrm{PbPb}$ collisions of various centralities are shown in figure 5 . The lower bound of $p_{\mathrm{T}}^{\text {jet }}$ is chosen based on the observed noise level for each centrality class, and the upper bound is driven by the amount of statistics. 


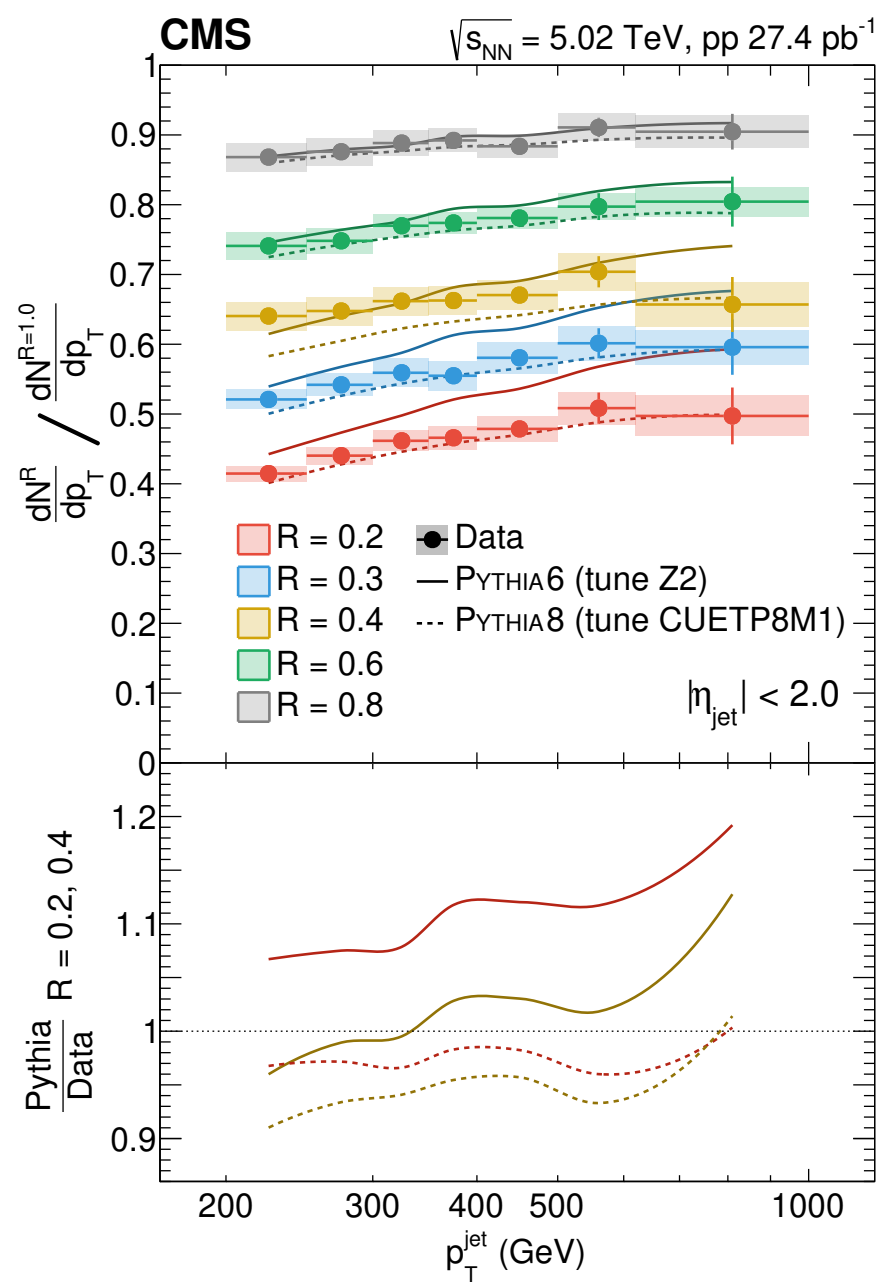

Figure 6. The spectra ratio for jets from pp collisions with $\left|\eta^{\text {jet }}\right|<2.0$ for $R=0.2-0.8$ with respect to $R=1.0$. The statistical uncertainty of data is shown as vertical lines, whereas the systematic uncertainties are shown as the shaded boxes. Markers for the data are placed at the bin centers. Comparisons with PYTHIA6 (solid line) and PYTHIA8 (dotted line) are plotted, along with ratios in the lower plot for $R=0.2$ and $R=0.4$.

The upper plot of figure 6 shows the ratio of spectra of jets with different radii in pp collisions, normalized to the spectrum for $R=1.0$. The number of jets with a given $p_{\mathrm{T}}^{\text {jet }}$ increases with the size of the jet cone. The increase of jet yield with $R$ becomes weaker at higher values of $p_{\mathrm{T}}^{\text {jet }}$ suggesting that jets become narrower as $p_{\mathrm{T}}^{\text {jet }}$ increases. Figure 6 also shows predictions using the PYTHIA6 and PYTHIA8 MC generators. Both generators capture the trends of the data but PYTHIA 8 is closer to the scale of the data. The lower plot of figure 6 shows the ratios of the jet spectra from PYTHIA to the data spectrum for $R=0.2$ and 0.4. For PYTHIA6 the ratio rises with $p_{\mathrm{T}}^{\text {jet }}$ for both values of $R$. The PYTHIA8 ratios show little dependence on $p_{\mathrm{T}}^{\text {jet }}$ and are generally closer to unity than those of PYTHIA6.

The $R_{\mathrm{AA}}$ factors compare $\mathrm{PbPb}$ data to the scaled pp reference. Figure 7 shows $R_{\mathrm{AA}}$, the ratio of $\mathrm{PbPb}$ data to a scaled $\mathrm{pp}$ reference, as functions of $p_{\mathrm{T}}^{\text {jet }}$, jet radius, and 


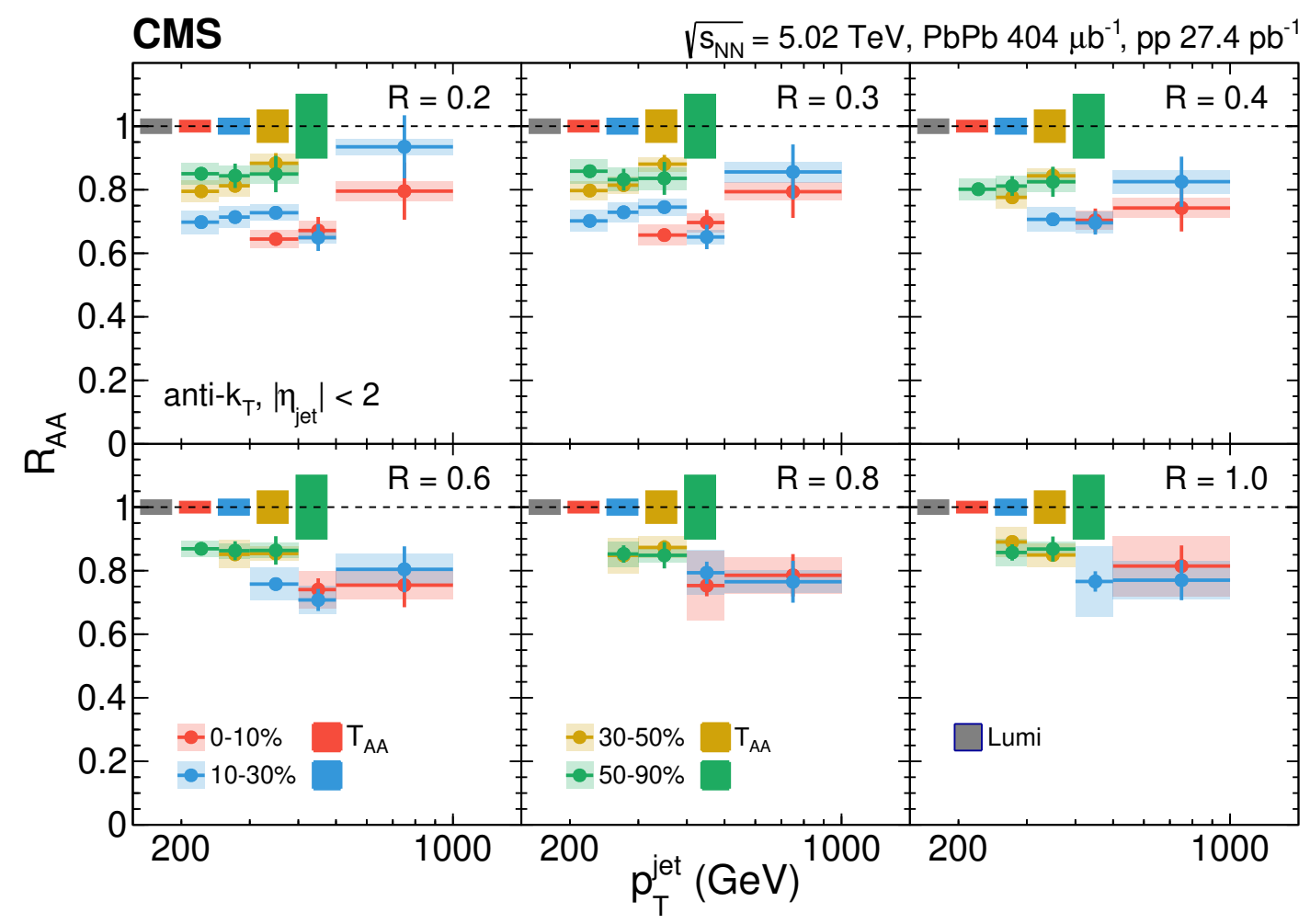

Figure 7. The $R_{\mathrm{AA}}$ for jets with $\left|\eta^{\mathrm{jet}}\right|<2.0$ as functions of $p_{\mathrm{T}}^{\text {jet }}$ for various $R$ and centrality classes. The statistical uncertainties are represented by vertical lines, and the systematic uncertainties by shaded boxes. The markers are placed at the bin centers. Global uncertainties (integrated luminosity for $\mathrm{pp}$ and $\left\langle T_{\mathrm{AA}}\right\rangle$ for $\mathrm{PbPb}$ data) are shown as colored boxes on the dashed line at $R_{\mathrm{AA}}=1$ and are not included in the shaded boxes around the points.

centrality. Systematic uncertainties related to the JES and JER cancel partially. The remaining systematic uncertainties are dominated by the uncertainties in the integrated luminosity, $\left\langle T_{\mathrm{AA}}\right\rangle$, and the JES uncertainty component from the UE.

For all values of $R, R_{\mathrm{AA}}$ for the most peripheral collisions (50-90\%) is independent of $p_{\mathrm{T}}^{\text {jet }}$ and consistent with unity after considering the $\left\langle T_{\mathrm{AA}}\right\rangle$ uncertainty. In the most central bin, a strong suppression of the $\mathrm{PbPb}$ data $(\approx 0.6-0.7)$ is observed, which is well outside the systematic uncertainties. However, there are hints of an increasing $R_{\mathrm{AA}}$ with $p_{\mathrm{T}}^{\text {jet }}$ for the smaller values of $R$ in the central bins, with values up to 0.8 for jets with $p_{\mathrm{T}}^{\text {jet }}>500 \mathrm{GeV}$.

To highlight the jet radius dependence of the jet $R_{\mathrm{AA}}$, the ratios of $R_{\mathrm{AA}}$ for a given $R$ with respect to $R=0.2$ are presented in figure 8 . This observable is particularly sensitive to the recovery of the quenched energy and the presence of the medium response [56]. For $400<p_{\mathrm{T}}^{\text {jet }}<500 \mathrm{GeV}$, the $R_{\mathrm{AA}}$ ratios are above unity and increase with $p_{\mathrm{T}}^{\text {jet }}$ in both the $0-10 \%$ and $10-30 \%$ centrality intervals. On the other hand, for $p_{\mathrm{T}}^{\text {jet }}>500 \mathrm{GeV}$, the $R_{\mathrm{AA}}^{R} / R_{\mathrm{AA}}^{R=0.2}$ is close to unity or slightly below it for the $0-10 \%$ and $10-30 \%$ centrality intervals, respectively. 


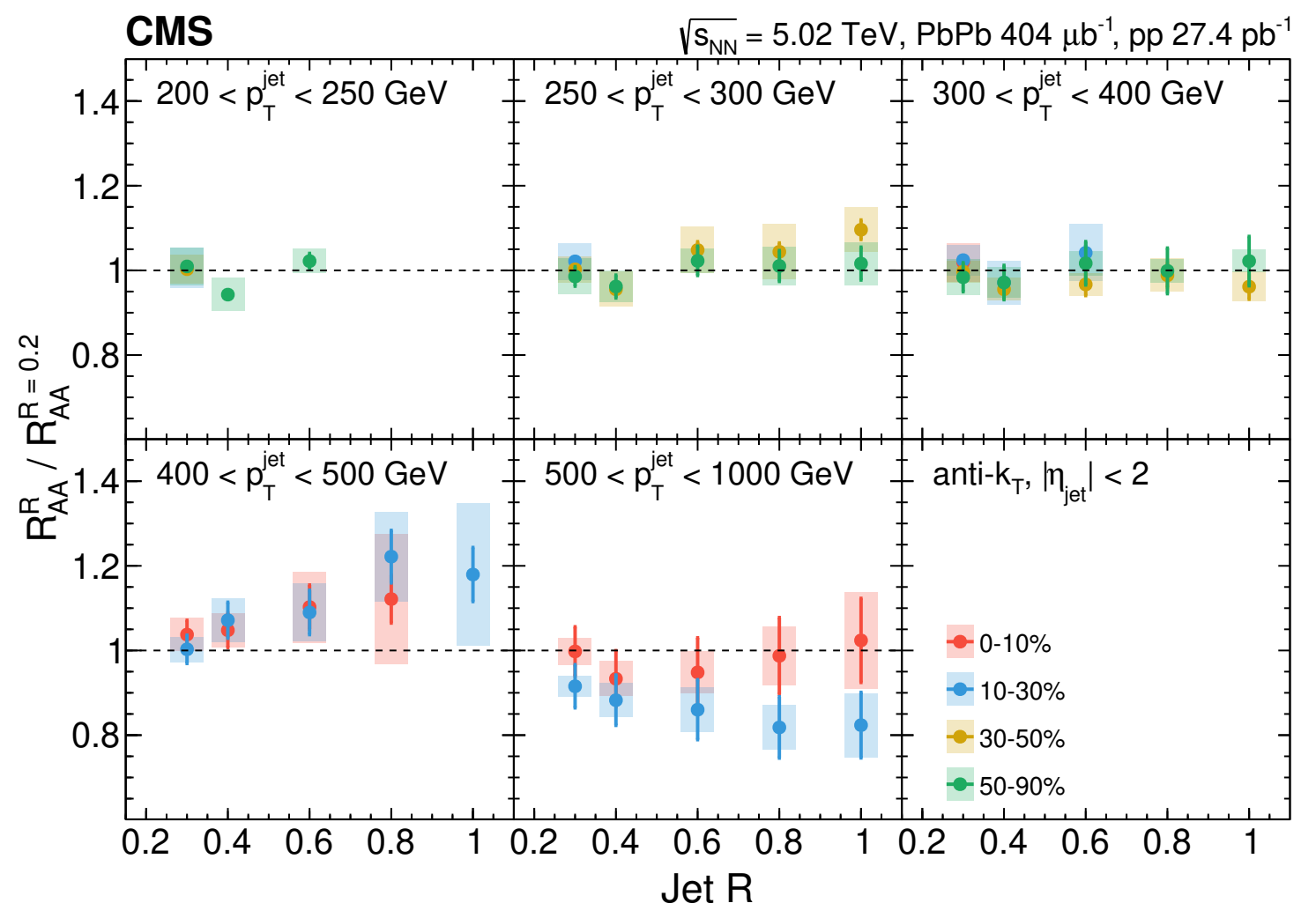

Figure 8. The $R_{\mathrm{AA}}$ ratio for jets with $\left|\eta^{\text {jet }}\right|<2.0$ as a function of $R$ for $R=0.3-1.0$ with respect to $R=0.2$, in various event centrality classes and $p_{\mathrm{T}}^{\text {jet }}$ ranges. The statistical uncertainties of data are shown as the vertical lines, whereas the systematic uncertainties are shown as the shaded boxes.

Figure 9 shows $R_{\mathrm{AA}}$ for $0-10 \%$ central $\mathrm{PbPb}$ collisions, as a function of $p_{\mathrm{T}}^{\text {jet }}$ for several $R$ values. As $p_{\mathrm{T}}^{\text {jet }}$ increases, $R_{\mathrm{AA}}$ increases. Also shown in figure 9 are predictions from the JEWEL [82] (v2.2.0) and PYQUEN [69] (v1.5.4) generators. The JEWEL predictions are made with (pink) and without (fuchsia) contributions from recoil particles (i.e. scattered medium particles). The predictions without recoil particles are in disagreement with the data, showing the importance of the medium response. The importance of recoil particles within JEWEL increases greatly as $R$ increases. For $R=1.0$ the predictions without recoil are a factor of four below the default mode with recoil. The JEWEL predictions with recoil are significantly below the data for $R=0.2$ but come increasingly close to the data as $R$ increases.

Predictions from PYQUEN are shown with (the default, shown in teal) and without (turquoise) medium-induced wide-angle radiation. The default PYQUEN generator overpredicts $R_{\mathrm{AA}}$ particularly for smaller values of $R$ and $p_{\mathrm{T}}^{\text {jet }}$. The inclusion of wide-angle radiation lowers the predictions for $R_{\mathrm{AA}}$ particularly for smaller $R$ sizes and brings the PYQUEN predictions closer to the data, showing the importance of the medium effects.

Figure 10 shows $R_{\mathrm{AA}}^{R} / R_{\mathrm{AA}}^{R=0.2}$ as a function of $R$ for several values of $p_{\mathrm{T}}^{\text {jet }}$. Monte Carlo predictions from the JEWEL and PYQUEN generators are also shown. For the data, $R_{\mathrm{AA}}^{R} / R_{\mathrm{AA}}^{R=0.2}$ has little dependence upon $R$ and is consistent with unity for all values of $p_{\mathrm{T}}^{\mathrm{jet}}$ 


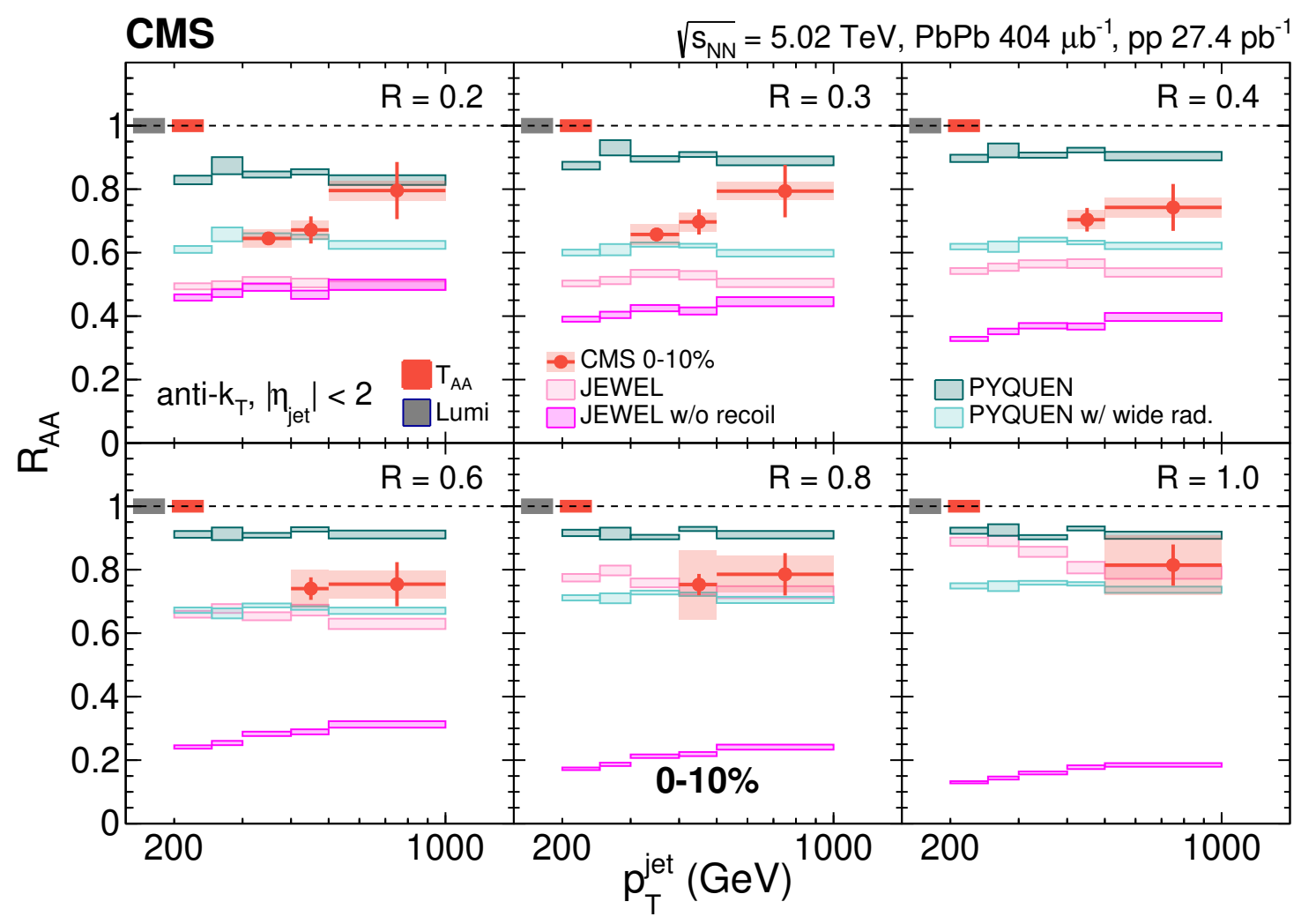

Figure 9. The $R_{\mathrm{AA}}$ for jets with $\left|\eta^{\text {jet }}\right|<2.0$, as a function of $p_{\mathrm{T}}^{\text {jet }}$, for various $R$ and $0-10 \%$ centrality class. The statistical uncertainties are represented by the vertical lines, while the systematic uncertainties are shown as the shaded boxes. The markers are placed at the bin centers. Global uncertainties (integrated luminosity for $\mathrm{pp}$ and $\left\langle T_{\mathrm{AA}}\right\rangle$ for $\mathrm{PbPb}$ collisions) are shown as the colored boxes on the dashed line at $R_{\mathrm{AA}}=1$ and are not included in the shaded bands around the points. The predictions from JEWEL (fuchsia and pink) and PYQUEN (teal and turquoise) generators, shown as colored boxes, are compared to the data.

for both the data and the PYQUEN predictions. The JEWEL model is unable to capture the $R$ dependence of $R_{\mathrm{AA}}^{R} / R_{\mathrm{AA}}^{R=0.2}$. For the predictions with recoil, $R_{\mathrm{AA}}^{R} / R_{\mathrm{AA}}^{R=0.2}$ increases as a function of $R$ but if recoil is ignored $R_{\mathrm{AA}}^{R} / R_{\mathrm{AA}}^{R=0.2}$ decreases with $R$.

Figure 11 shows a comparison of several models to $R_{\mathrm{AA}}$ as functions of $p_{\mathrm{T}}^{\text {jet }}$ and $R$. The HYBRID model [56] combines a perturbative description of the weakly coupled physics of jet production and evolution, with a gauge/gravity duality description of the strongly coupled dynamics of the medium, and the soft-gluon exchanges between the jet and medium. As the jet passes through and deposits energy into the hydrodynamic medium, a wake is left behind the jet. The HYBRID model (dark orange) tends to under-predict $R_{\mathrm{AA}}$ at high $p_{\mathrm{T}}^{\text {jet }}$. Calculations without a wake (brown) and with only the positive contribution of the wake (yellow) are also shown. These two are not physical and are included here only for better understanding of the effect of the wake contribution. The effect of the wake is more important at large $R$ and lower $p_{\mathrm{T}}^{\mathrm{jet}}$. 


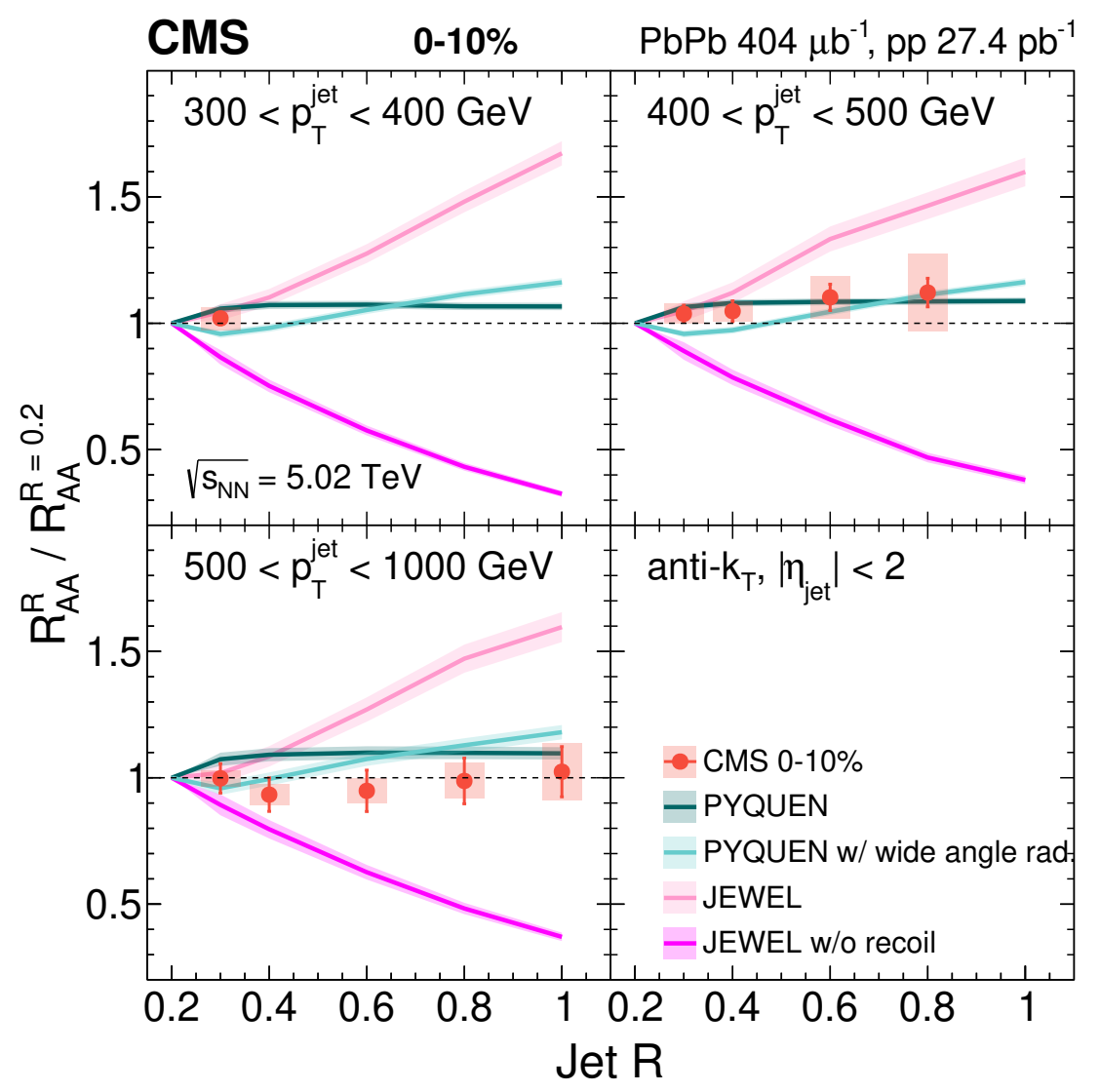

Figure 10. The $R_{\mathrm{AA}}$ ratio for jets with $\left|\eta^{\text {jet }}\right|<2.0$ as a function of $R$ for $R=0.3-1.0$ with respect to $R=0.2$, in various $p_{\mathrm{T}}^{\text {jet }}$ ranges for the $0-10 \%$ centrality class. The statistical uncertainties of data are shown as the vertical lines, whereas the systematic uncertainties are shown as the shaded boxes. The width of the boxes carries no meaning. The predictions from JEWEL (fuchsia and pink) and PYQUEN (teal and turquoise) generators, shown with the colored bands, are compared to the data.

In the Linear Boltzmann Transport (LBT) model [83], the effects of recoil thermal partons and their propagation in the dense medium are described by a $3+1 \mathrm{D}$ viscous relativistic hydrodynamic model. Predictions from LBT are shown in figure 11 with and without the medium response. It is clear that the medium response becomes more and more dominant as the size of the jet cone increases. A similar effect is seen for the jetcoupled fluid model $[52,84,85]$ CCNU. Although predictions are only available for a limited $p_{\mathrm{T}}^{\text {jet }}$ range, it is clear from comparing the blue and violet points in figure 11 that the hydrodynamic component of $\mathrm{CCNU}$ becomes increasingly important with increasing $R$.

The predictions from MARTINI [86] (Modular Algorithm for Relativistic Treatment of Heavy IoN Interactions) are shown as purple boxes in figure 11. The model follows a hybrid approach where it embeds the high energy parton into an evolving hydrodynamic medium, and the shower evolution of the jet is modified following the McGill-AMY formalism [8791]. The MARTINI generator predicts a larger increase of jet $R_{\mathrm{AA}}$ ratio as a function of $R$ than what is observed in data. 


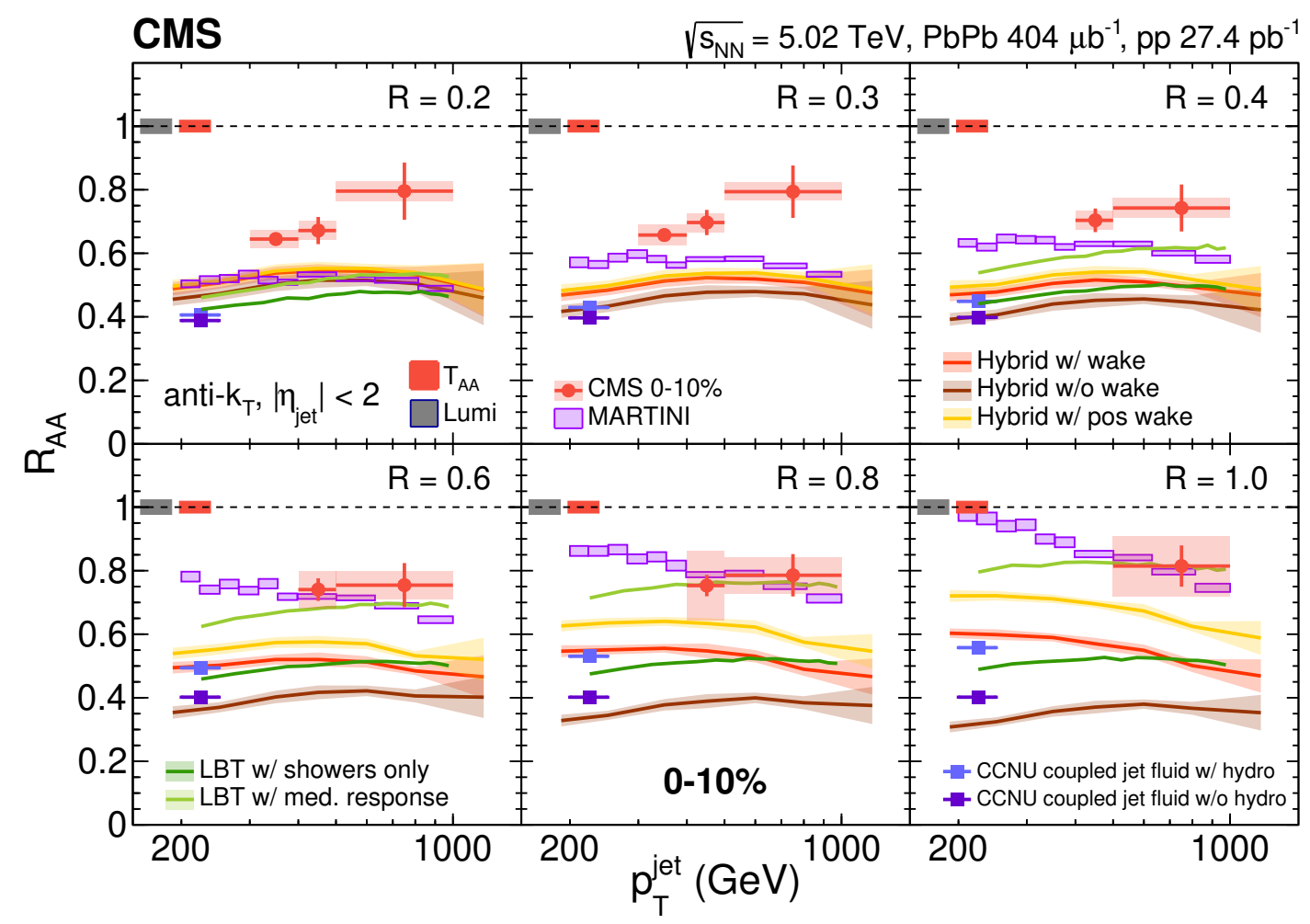

Figure 11. The $R_{\mathrm{AA}}$ for jets with $\left|\eta^{\text {jet }}\right|<2.0$, as a function of $p_{\mathrm{T}}^{\text {jet }}$, for various $R$ values and the $0-10 \%$ centrality class. The statistical uncertainties are represented by vertical lines, while the systematic uncertainties are shown as shaded boxes. The markers are placed at the bin centers. Global uncertainties (integrated luminosity for pp and $\left\langle T_{\mathrm{AA}}\right\rangle$ for $\mathrm{PbPb}$ collisions) are shown as the colored boxes on the dashed line at $R_{\mathrm{AA}}=1$ and are not included in the shaded bands around the points. The predictions from HYBRID (dark orange, brown and yellow), MARTINI (purple), LBT (lime and dark green), and CCNU (blue and violet) models, shown as the colored boxes and bands, are compared to the data.

From figure 11, it is striking that, for the small jet radius $R=0.2, R_{\mathrm{AA}}$ rises with $p_{\mathrm{T}}^{\text {jet }}$ but the Hybrid, LBT, CCNU and MarTini models are all flat in $p_{\mathrm{T}}^{\text {jet }}$. For all these models, hydrodynamic or medium effects become increasingly important as $R$ increases and are indeed dominant for $R=1.0$.

Figure 12 shows $R_{\mathrm{AA}}^{R} / R_{\mathrm{AA}}^{R=0.2}$ as a function of $R$, for several values of $p_{\mathrm{T}}^{\text {jet }}$. Monte Carlo predictions from the HYBRID, MARTINI, and LBT generators are also shown. The HYBRID model (orange) is able to describe the data. However if the wake contribution is ignored (brown) the model gives a different trend. The MARTINI model (purple) predicts that $R_{\mathrm{AA}}^{R} / R_{\mathrm{AA}}^{R=0.2}$ should increase with $R$ in contrast to the data. The default LBT model (lime) is consistent with the data but LBT with showers only and no medium response (dark green) overpredicts $R_{\mathrm{AA}}^{R} / R_{\mathrm{AA}}^{R=0.2}$. Some of the models which correctly predict the trend of $R_{\mathrm{AA}}^{R} / R_{\mathrm{AA}}^{R=0.2}$ are off in the $R_{\mathrm{AA}}$, as can be seen in figure 11 . 


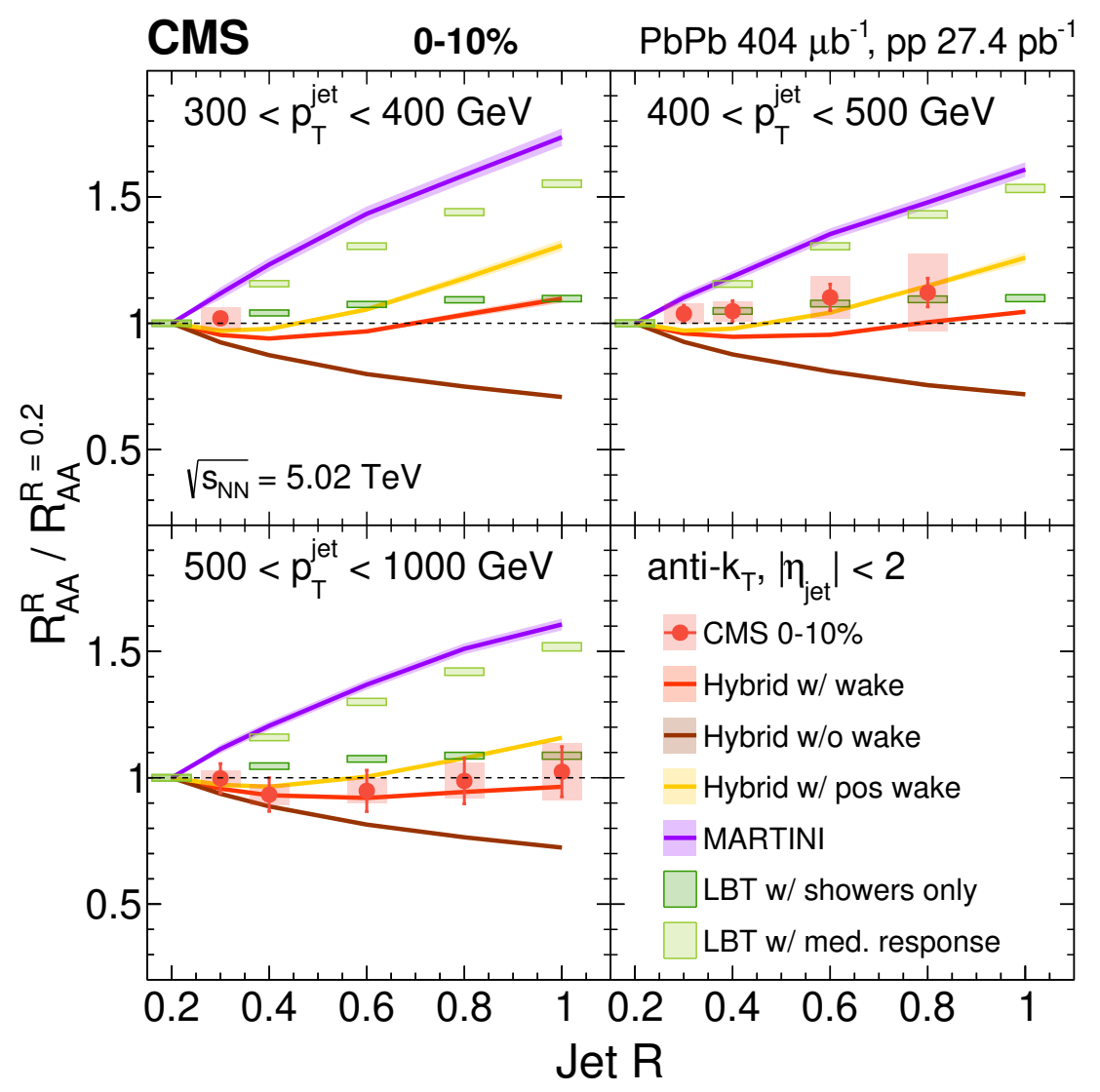

Figure 12. The double ratio $R_{\mathrm{AA}}^{R} / R_{\mathrm{AA}}^{R=0.2}$ for jets with $\left|\eta^{\text {jet }}\right|<2.0$, as a function of $R$, for $R=0.3^{-}$ 1.0 with respect to $R=0.2$, in various $p_{\mathrm{T}}^{\text {jet }}$ ranges for the $0-10 \%$ centrality class. The statistical uncertainties of data are shown as the vertical lines, whereas the systematic uncertainties are shown as the shaded boxes. The width of the boxes carries no meaning. The predictions from the HYBRID (dark orange, brown and yellow), MARTINI (purple), and LBT (lime and dark green) models are compared to the data as colored bands.

The same data in figures 11 and 12 are also compared to additional models. Figure 13 shows $R_{\mathrm{AA}}$ vs. $p_{\mathrm{T}}^{\text {jet }}$ for several values of $R$ and for the top $0-10 \%$ centrality class as well as several predictions from generators and analytic calculations. The gray boxes in figure 13 are predictions from a jet factorization model based on a phenomenological approach to establish QCD factorization of jet cross sections in heavy ion collisions [92]. Mediummodified jet functions are extracted from jet nuclear modification factors at smaller jet distance parameter values $(R=0.2$ and 0.4$)$ and predictions are made for larger distance parameter values. At $R<0.4$, the data are described reasonably well by the factorization model. However, the model tends to underpredict $R_{\mathrm{AA}}$ at larger $R$ values. The data are also compared to the coherent antenna BDMPS calculations [93] (orange), which is an analytical approach that resums multiple emissions to leading-logarithmic accuracy including both radiative energy loss and color coherence effects [94-96]. The predictions are in general agreement with the $R_{\mathrm{AA}}$ data. 


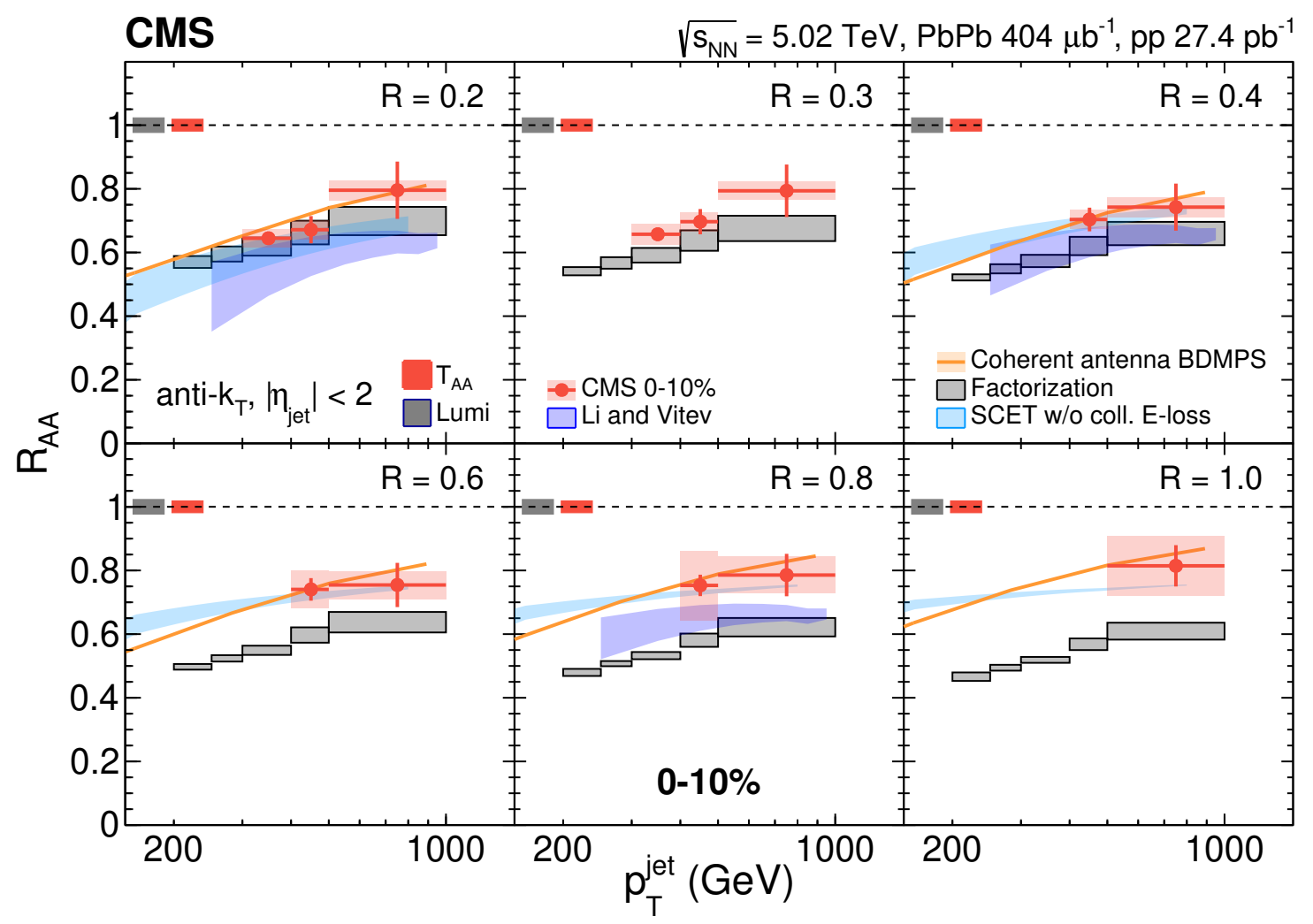

Figure 13. The $R_{\mathrm{AA}}$ for jets with $\left|\eta^{\text {jet }}\right|<2.0$, as a function of $p_{\mathrm{T}}^{\text {jet }}$, for various $R$ values and 0-10\% centrality class. The statistical uncertainties are represented by the vertical lines, while the systematic uncertainties are shown as the shaded boxes. The markers are placed at the bin centers. Global uncertainties (integrated luminosity for pp and $\left\langle T_{\mathrm{AA}}\right\rangle$ for $\mathrm{PbPb}$ collisions) are shown as the colored boxes on the dashed line at $R_{\mathrm{AA}}=1$ and are not included in the shaded bands around the points. The calculations from SCET (sky blue and navy blue), coherent antenna BDMPs (orange) and jet factorization (gray) formalisms are compared to the data, shown as the colored boxes and bands.

Finally, calculations based on a soft collinear effective theory with Glauber gluon interactions SCET [50], are also compared to the data. The SCET calculations with collisional energy loss $[97,98]$ (navy blue) are slightly below the $R_{\mathrm{AA}}$ measurements while those without collisional energy loss (sky blue) are consistent with the data.

Figure 14 shows $R_{\mathrm{AA}}^{R} / R_{\mathrm{AA}}^{R=0.2}$ vs. $R$ for several values of $p_{\mathrm{T}}^{\text {jet }}$ together with predictions from the SCET, BDMPS and jet factorization models. The BDMPS (orange) and SCET predictions (sky blue and navy blue) are consistent with the data but the factorization calculations (gray) decrease with $R$ in contrast to the data.

\section{Summary}

Measurements of jet nuclear modification factors based on proton-proton and lead-lead collisions at $\sqrt{s_{\mathrm{NN}}}=5.02 \mathrm{TeV}$ are presented. For the first time, jet spectra measurements are extended to large area jets, with a anti- $k_{\mathrm{T}}$ distance parameter $R$ up to 1.0. For the most 


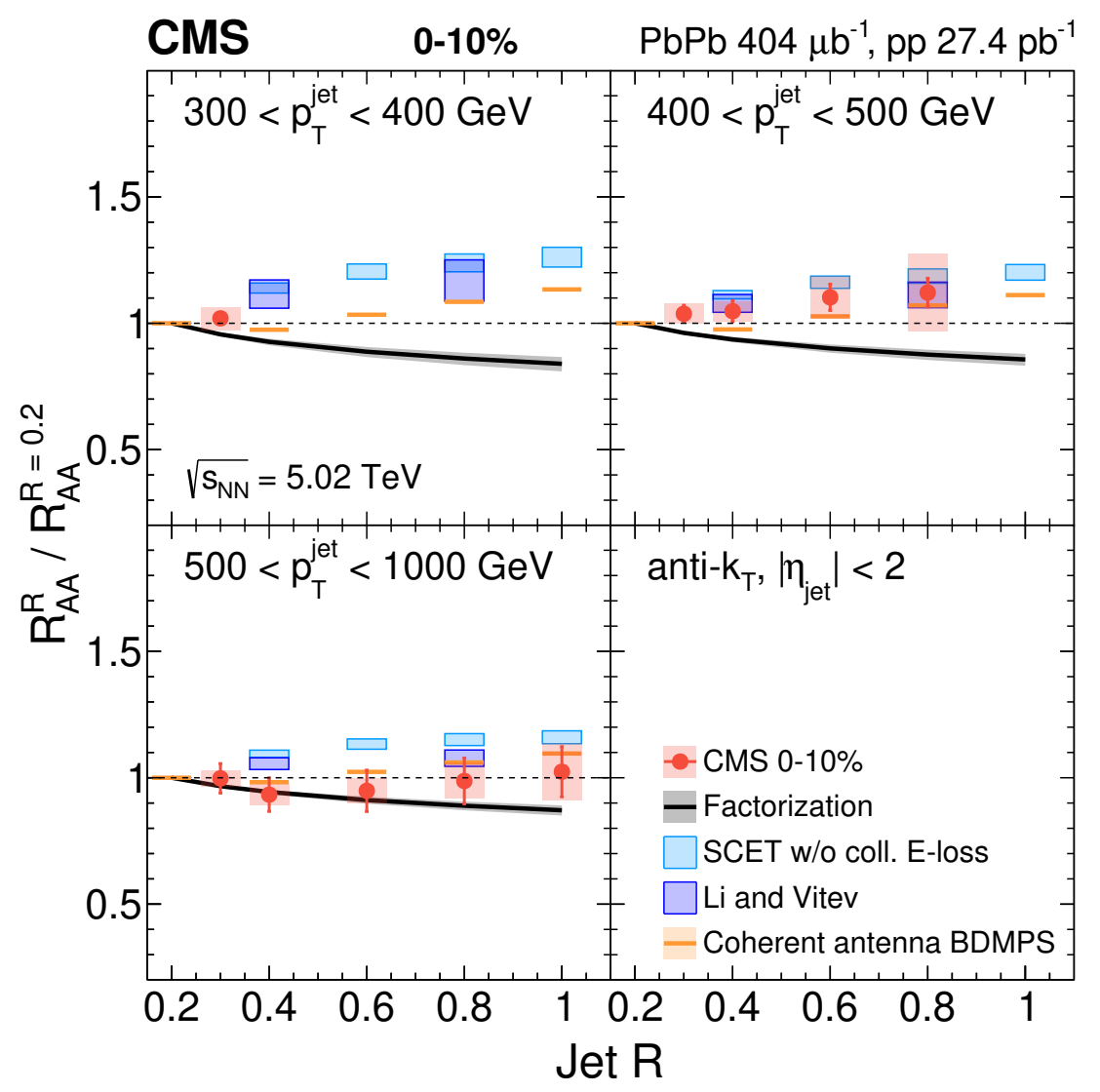

Figure 14. The double ratio $R_{\mathrm{AA}}^{R} / R_{\mathrm{AA}}^{R=0.2}$ for jets with $\left|\eta^{\text {jet }}\right|<2.0$ as a function of $R$ for $R=0.3^{-}$ 1.0 with respect to $R=0.2$, in various $p_{\mathrm{T}}^{\text {jet }}$ ranges for the $0-10 \%$ centrality class. The statistical uncertainties of data are shown as the vertical lines, whereas the systematic uncertainties are shown as the shaded boxes. The width of the boxes carries no meaning. The calculations based from SCET (sky blue and navy blue), coherent antenna BDMPs (orange) and, jet factorization (gray) formalisms, shown with the colored bands and boxes, are compared to the data.

central $\mathrm{PbPb}$ collisions, a strong suppression is observed for jets with high transverse momentum reconstructed with all distance parameters. Predictions from quenched jet event generators, theoretical models, and analytical calculations are compared to these results. The new data place further constraints on the underlying jet quenching mechanisms. While state of the art models have made important progress, significant tension remains in view of the large area jet data presented here.

\section{Acknowledgments}

We congratulate our colleagues in the CERN accelerator departments for the excellent performance of the LHC and thank the technical and administrative staffs at CERN and at other CMS institutes for their contributions to the success of the CMS effort. In addition, we gratefully acknowledge the computing centers and personnel of the Worldwide 
LHC Computing Grid and other centers for delivering so effectively the computing infrastructure essential to our analyses. Finally, we acknowledge the enduring support for the construction and operation of the LHC, the CMS detector, and the supporting computing infrastructure provided by the following funding agencies: BMBWF and FWF (Austria); FNRS and FWO (Belgium); CNPq, CAPES, FAPERJ, FAPERGS, and FAPESP (Brazil); MES (Bulgaria); CERN; CAS, MoST, and NSFC (China); COLCIENCIAS (Colombia); MSES and CSF (Croatia); RIF (Cyprus); SENESCYT (Ecuador); MoER, ERC PUT and ERDF (Estonia); Academy of Finland, MEC, and HIP (Finland); CEA and CNRS/IN2P3 (France); BMBF, DFG, and HGF (Germany); GSRT (Greece); NKFIA (Hungary); DAE and DST (India); IPM (Iran); SFI (Ireland); INFN (Italy); MSIP and NRF (Republic of Korea); MES (Latvia); LAS (Lithuania); MOE and UM (Malaysia); BUAP, CINVESTAV, CONACYT, LNS, SEP, and UASLP-FAI (Mexico); MOS (Montenegro); MBIE (New Zealand); PAEC (Pakistan); MSHE and NSC (Poland); FCT (Portugal); JINR (Dubna); MON, RosAtom, RAS, RFBR, and NRC KI (Russia); MESTD (Serbia); SEIDI, CPAN, PCTI, and FEDER (Spain); MOSTR (Sri Lanka); Swiss Funding Agencies (Switzerland); MST (Taipei); ThEPCenter, IPST, STAR, and NSTDA (Thailand); TUBITAK and TAEK (Turkey); NASU (Ukraine); STFC (United Kingdom); DOE and NSF (U.S.A.).

Individuals have received support from the Marie-Curie program and the European Research Council and Horizon 2020 Grant, contract Nos. 675440, 724704, 752730, and 765710 (European Union); the Leventis Foundation; the Alfred P. Sloan Foundation; the Alexander von Humboldt Foundation; the Belgian Federal Science Policy Office; the Fonds pour la Formation à la Recherche dans l'Industrie et dans l'Agriculture (FRIABelgium); the Agentschap voor Innovatie door Wetenschap en Technologie (IWT-Belgium); the F.R.S.-FNRS and FWO (Belgium) under the "Excellence of Science - EOS" be.h project n. 30820817; the Beijing Municipal Science \& Technology Commission, No. Z191100007219010; the Ministry of Education, Youth and Sports (MEYS) of the Czech Republic; the Deutsche Forschungsgemeinschaft (DFG), under Germany's Excellence Strategy — EXC 2121 "Quantum Universe" — 390833306, and under project number 400140256 GRK2497; the Lendület ("Momentum") Program and the János Bolyai Research Scholarship of the Hungarian Academy of Sciences, the New National Excellence Program ÚNKP, the NKFIA research grants 123842, 123959, 124845, 124850, 125105, 128713, 128786, and 129058 (Hungary); the Council of Science and Industrial Research, India; the Ministry of Science and Higher Education and the National Science Center, contracts Opus 2014/15/B/ST2/03998 and 2015/19/B/ST2/02861 (Poland); the National Priorities Research Program by Qatar National Research Fund; the Ministry of Science and Higher Education, project no. 0723-2020-0041 (Russia); the Programa Estatal de Fomento de la Investigación Científica y Técnica de Excelencia María de Maeztu, grant MDM-20150509 and the Programa Severo Ochoa del Principado de Asturias; the Thalis and Aristeia programs cofinanced by EU-ESF and the Greek NSRF; the Rachadapisek Sompot Fund for Postdoctoral Fellowship, Chulalongkorn University and the Chulalongkorn Academic into Its 2nd Century Project Advancement Project (Thailand); the Kavli Foundation; the Nvidia Corporation; the SuperMicro Corporation; the Welch Foundation, contract C-1845; and the Weston Havens Foundation (U.S.A.). 
Open Access. This article is distributed under the terms of the Creative Commons Attribution License (CC-BY 4.0), which permits any use, distribution and reproduction in any medium, provided the original author(s) and source are credited.

\section{References}

[1] F. Karsch, The phase transition to the quark gluon plasma: recent results from lattice calculations, Nucl. Phys. A 590 (1995) 367C [hep-lat/9503010] [INSPIRE].

[2] J.D. Bjorken, Highly relativistic nucleus-nucleus collisions: the central rapidity region, Phys. Rev. D 27 (1983) 140 [inSPIRE].

[3] J.E. Bernhard, J.S. Moreland, S.A. Bass, J. Liu and U. Heinz, Applying Bayesian parameter estimation to relativistic heavy-ion collisions: simultaneous characterization of the initial state and quark-gluon plasma medium, Phys. Rev. C 94 (2016) 024907 [arXiv:1605.03954] [INSPIRE].

[4] J.D. Bjorken, Energy loss of energetic partons in quark-gluon plasma: possible extinction of high $p_{\mathrm{T}}$ jets in hadron-hadron collisions, FNAL public document FERMILAB-PUB-82-059-THY, FNAL, Batavia, IL, U.S.A. (1982).

[5] M. Gyulassy and M. Plümer, Jet quenching in dense matter, Phys. Lett. B 243 (1990) 432 [INSPIRE].

[6] X.-N. Wang and M. Gyulassy, Gluon shadowing and jet quenching in A $A$ collisions at $\sqrt{s}=200 \mathrm{GeV}$, Phys. Rev. Lett. 68 (1992) 1480 [inSPIRE].

[7] R. Baier, Y.L. Dokshitzer, A.H. Mueller, S. Peigne and D. Schiff, Radiative energy loss and $p_{\mathrm{T}}$ broadening of high-energy partons in nuclei, Nucl. Phys. B 484 (1997) 265 [hep-ph/9608322] [INSPIRE].

[8] B.G. Zakharov, Radiative energy loss of high-energy quarks in finite size nuclear matter and quark-gluon plasma, JETP Lett. 65 (1997) 615 [hep-ph/9704255] [INSPIRE].

[9] D.A. Appel, Jets as a probe of quark-gluon plasmas, Phys. Rev. D 33 (1986) 717 [INSPIRE].

[10] J.P. Blaizot and L.D. McLerran, Jets in expanding quark-gluon plasmas, Phys. Rev. D 34 (1986) 2739 [INSPIRE].

[11] JET collaboration, Extracting the jet transport coefficient from jet quenching in high-energy heavy-ion collisions, Phys. Rev. C 90 (2014) 014909 [arXiv: 1312.5003] [INSPIRE].

[12] H. Liu, K. Rajagopal and U.A. Wiedemann, Calculating the jet quenching parameter from AdS/CFT, Phys. Rev. Lett. 97 (2006) 182301 [hep-ph/0605178] [INSPIRE].

[13] J. Casalderrey-Solana and C.A. Salgado, Introductory lectures on jet quenching in heavy ion collisions, Acta Phys. Polon. B 38 (2007) 3731 [arXiv:0712.3443] [inSPIRE].

[14] D. d'Enterria, Jet quenching, Landolt-Bornstein 23 (2010) 471 [arXiv:0902.2011] [INSPIRE].

[15] U.A. Wiedemann, Jet quenching in heavy ion collisions, Landolt-Bornstein 23 (2010) 521 [arXiv: 0908.2306] [INSPIRE].

[16] A. Majumder and M. Van Leeuwen, The theory and phenomenology of perturbative QCD based jet quenching, Prog. Part. Nucl. Phys. 66 (2011) 41 [arXiv:1002.2206] [InSPIRE].

[17] G.-Y. Qin and X.-N. Wang, Jet quenching in high-energy heavy-ion collisions, Int. J. Mod. Phys. E 24 (2015) 1530014 [arXiv: 1511.00790] [InSPIRE]. 
[18] S. Cao and X.-N. Wang, Jet quenching and medium response in high-energy heavy-ion collisions: a review, Rept. Prog. Phys. 84 (2021) 024301 [arXiv: 2002.04028] [INSPIRE].

[19] PHENIX collaboration, Formation of dense partonic matter in relativistic nucleus-nucleus collisions at RHIC: experimental evaluation by the PHENIX collaboration, Nucl. Phys. A 757 (2005) 184 [nucl-ex/0410003] [INSPIRE].

[20] STAR collaboration, Experimental and theoretical challenges in the search for the quark gluon plasma: the STAR collaboration's critical assessment of the evidence from RHIC collisions, Nucl. Phys. A 757 (2005) 102 [nucl-ex/0501009] [INSPIRE].

[21] PHOBOS collaboration, The PHOBOS perspective on discoveries at RHIC, Nucl. Phys. A 757 (2005) 28 [nucl-ex/0410022] [INSPIRE].

[22] BRAHMS collaboration, Quark gluon plasma and color glass condensate at RHIC? The perspective from the BRAHMS experiment, Nucl. Phys. A 757 (2005) 1 [nucl-ex/0410020] [INSPIRE].

[23] PHENIX collaboration, Transverse momentum and centrality dependence of dihadron correlations in $A u+A u$ collisions at $\sqrt{s_{N N}}=200 \mathrm{GeV}$ : jet-quenching and the response of partonic matter, Phys. Rev. C $\mathbf{7 7}$ (2008) 011901 [arXiv: 0705.3238] [INSPIRE].

[24] CMS collaboration, Study of high- $p_{\mathrm{T}}$ charged particle suppression in PbPb compared to $p p$ collisions at $\sqrt{s_{N N}}=2.76 \mathrm{TeV}$, Eur. Phys. J. C 72 (2012) 1945 [arXiv:1202.2554] [INSPIRE].

[25] ATLAS collaboration, Measurement of charged-particle spectra in $\mathrm{Pb}+\mathrm{Pb}$ collisions at $\sqrt{s_{N N}}=2.76 \mathrm{TeV}$ with the ATLAS detector at the LHC, JHEP 09 (2015) 050 [arXiv: 1504.04337] [INSPIRE].

[26] ALICE collaboration, Suppression of charged particle production at large transverse momentum in central Pb-Pb collisions at $\sqrt{s_{N N}}=2.76 \mathrm{TeV}$, Phys. Lett. B 696 (2011) 30 [arXiv: 1012.1004] [INSPIRE].

[27] M. Cacciari, G.P. Salam and G. Soyez, The anti- $k_{t}$ jet clustering algorithm, JHEP 04 (2008) 063 [arXiv:0802.1189] [inSPIRE].

[28] ATLAS collaboration, Observation of a centrality-dependent dijet asymmetry in lead-lead collisions at $\sqrt{s_{N N}}=2.77 \mathrm{TeV}$ with the ATLAS detector at the LHC, Phys. Rev. Lett. 105 (2010) 252303 [arXiv: 1011.6182] [INSPIRE].

[29] CMS collaboration, Observation and studies of jet quenching in $\mathrm{PbPb}$ collisions at nucleon-nucleon center-of-mass energy $=2.76 \mathrm{TeV}$, Phys. Rev. C 84 (2011) 024906 [arXiv:1102.1957] [INSPIRE].

[30] STAR collaboration, Dijet imbalance measurements in Au+Au and pp collisions at $\sqrt{s_{N N}}=200 \mathrm{GeV}$ at STAR, Phys. Rev. Lett. 119 (2017) 062301 [arXiv:1609.03878] [INSPIRE].

[31] ATLAS collaboration, Measurements of the nuclear modification factor for jets in $\mathrm{Pb}+\mathrm{Pb}$ collisions at $\sqrt{s_{N N}}=2.76 \mathrm{TeV}$ with the ATLAS detector, Phys. Rev. Lett. 114 (2015) 072302 [arXiv:1411.2357] [INSPIRE].

[32] ALICE collaboration, Measurement of jet suppression in central $\mathrm{Pb}$ - $\mathrm{Pb}$ collisions at $\sqrt{s_{N N}}=2.76 \mathrm{TeV}$, Phys. Lett. B 746 (2015) 1 [arXiv:1502.01689] [INSPIRE].

[33] CMS collaboration, Measurement of inclusive jet cross sections in pp and $\mathrm{PbPb}$ collisions at $\sqrt{s_{N N}}=2.76 \mathrm{TeV}$, Phys. Rev. C 96 (2017) 015202 [arXiv: 1609.05383] [INSPIRE]. 
[34] STAR collaboration, Measurement of inclusive charged-particle jet production in $A u+A u$ collisions at $\sqrt{s_{N N}}=200 \mathrm{GeV}$, Phys. Rev. C 102 (2020) 054913 [arXiv:2006.00582] [INSPIRE].

[35] ATLAS collaboration, Measurement of the nuclear modification factor for inclusive jets in $P b+P b$ collisions at $\sqrt{s_{N N}}=5.02 \mathrm{TeV}$ with the ATLAS detector, Phys. Lett. B 790 (2019) 108 [arXiv: 1805.05635$]$ [INSPIRE].

[36] ALICE collaboration, Measurements of inclusive jet spectra in pp and central $\mathrm{Pb}-\mathrm{Pb}$ collisions at $\sqrt{s_{N N}}=5.02 \mathrm{TeV}$, Phys. Rev. C 101 (2020) 034911 [arXiv:1909.09718] [INSPIRE].

[37] ALICE collaboration, Measurement of jet quenching with semi-inclusive hadron-jet distributions in central Pb-Pb collisions at $\sqrt{s_{N N}}=2.76 \mathrm{TeV}$, JHEP 09 (2015) 170 [arXiv: 1506.03984] [INSPIRE].

[38] CMS collaboration, Studies of jet quenching using isolated-photon+jet correlations in $\mathrm{PbPb}$ and pp collisions at $\sqrt{s_{N N}}=2.76 \mathrm{TeV}$, Phys. Lett. B 718 (2013) 773 [arXiv:1205.0206] [INSPIRE].

[39] CMS collaboration, Study of jet quenching with isolated-photon+jet correlations in $\mathrm{PbPb}$ and pp collisions at $\sqrt{s_{N N}}=5.02$ TeV, Phys. Lett. B 785 (2018) 14 [arXiv:1711.09738] [INSPIRE].

[40] CMS collaboration, Measurement of jet fragmentation in $\mathrm{PbPb}$ and pp collisions at $\sqrt{s_{N N}}=2.76 \mathrm{TeV}$, Phys. Rev. C 90 (2014) 024908 [arXiv: 1406.0932] [INSPIRE].

[41] ATLAS collaboration, Measurement of jet fragmentation in $\mathrm{Pb}+\mathrm{Pb}$ and $p p$ collisions at $\sqrt{s_{N N}}=2.76 \mathrm{TeV}$ with the ATLAS detector at the LHC, Eur. Phys. J. C $\mathbf{7 7}$ (2017) 379 [arXiv: 1702.00674] [INSPIRE].

[42] CMS collaboration, Observation of medium-induced modifications of jet fragmentation in $\mathrm{Pb}$-Pb collisions at $\sqrt{s_{N N}}=5.02 \mathrm{TeV}$ using isolated photon-tagged jets, Phys. Rev. Lett. 121 (2018) 242301 [arXiv:1801.04895] [INSPIRE].

[43] CMS collaboration, Measurement of transverse momentum relative to dijet systems in $\mathrm{PbPb}$ and pp collisions at $\sqrt{s_{N N}}=2.76 \mathrm{TeV}, \mathrm{JHEP} 01$ (2016) 006 [arXiv:1509.09029] [INSPIRE].

[44] CMS collaboration, Decomposing transverse momentum balance contributions for quenched jets in PbPb collisions at $\sqrt{s_{N N}}=2.76 \mathrm{TeV}$, JHEP 11 (2016) 055 [arXiv:1609.02466] [INSPIRE].

[45] CMS collaboration, Modification of jet shapes in PbPb collisions at $\sqrt{s_{N N}}=2.76 \mathrm{TeV}$, Phys. Lett. B 730 (2014) 243 [arXiv:1310.0878] [INSPIRE].

[46] CMS collaboration, Jet properties in PbPb and pp collisions at $\sqrt{s_{N N}}=5.02 \mathrm{TeV}$, JHEP 05 (2018) 006 [arXiv : 1803.00042] [INSPIRE].

[47] CMS collaboration, Jet shapes of isolated photon-tagged jets in $\mathrm{Pb}-\mathrm{Pb}$ and $\mathrm{pp}$ collisions at $\sqrt{s_{N N}}=5.02 \mathrm{TeV}$, Phys. Rev. Lett. 122 (2019) 152001 [arXiv:1809.08602] [INSPIRE].

[48] ALICE collaboration, Measurement of jet radial profiles in $\mathrm{Pb}-\mathrm{Pb}$ collisions at $\sqrt{s_{N N}}=2.76 \mathrm{TeV}$, Phys. Lett. B 796 (2019) 204 [arXiv:1904.13118] [INSPIRE].

[49] STAR collaboration, Measurements of jet quenching with semi-inclusive hadron+jet distributions in Au+Au collisions at $\sqrt{s_{N N}}=200 \mathrm{GeV}$, Phys. Rev. C 96 (2017) 024905 [arXiv: 1702.01108] [INSPIRE]. 
[50] Y.-T. Chien and I. Vitev, Towards the understanding of jet shapes and cross sections in heavy ion collisions using soft-collinear effective theory, JHEP 05 (2016) 023 [arXiv: 1509.07257] [INSPIRE].

[51] N. Armesto, L. Cunqueiro and C.A. Salgado, Q-PYTHIA: a medium-modified implementation of final state radiation, Eur. Phys. J. C 63 (2009) 679 [arXiv:0907.1014] [INSPIRE].

[52] Y. Tachibana, N.-B. Chang and G.-Y. Qin, Full jet in quark-gluon plasma with hydrodynamic medium response, Phys. Rev. C 95 (2017) 044909 [arXiv:1701.07951] [INSPIRE].

[53] Z. Hulcher, D. Pablos and K. Rajagopal, Resolution effects in the hybrid strong/weak coupling model, JHEP 03 (2018) 010 [arXiv:1707.05245] [INSPIRE].

[54] C. Loizides, J. Kamin and D. d'Enterria, Improved Monte Carlo Glauber predictions at present and future nuclear colliders, Phys. Rev. C 97 (2018) 054910 [Erratum ibid. 99 (2019) 019901] [arXiv:1710.07098] [INSPIRE].

[55] M.L. Miller, K. Reygers, S.J. Sanders and P. Steinberg, Glauber modeling in high energy nuclear collisions, Ann. Rev. Nucl. Part. Sci. 57 (2007) 205 [nucl-ex/0701025] [InSPIRE].

[56] D. Pablos, Jet suppression from a small to intermediate to large radius, Phys. Rev. Lett. 124 (2020) 052301 [arXiv: 1907.12301] [INSPIRE].

[57] CMS collaboration, Performance of photon reconstruction and identification with the CMS detector in proton-proton collisions at $\sqrt{s}=8 \mathrm{TeV}, 2015$ JINST $10 \mathrm{P} 08010$ [arXiv: 1502.02702] [INSPIRE].

[58] CMS collaboration, Particle-flow reconstruction and global event description with the CMS detector, 2017 JINST 12 P10003 [arXiv:1706.04965] [INSPIRE].

[59] CMS collaboration, Description and performance of track and primary-vertex reconstruction with the CMS tracker, 2014 JINST 9 P10009 [arXiv:1405.6569] [INSPIRE].

[60] CMS collaboration, The CMS trigger system, 2017 JINST 12 P01020 [arXiv:1609.02366] [INSPIRE].

[61] CMS collaboration, The CMS experiment at the CERN LHC, 2008 JINST 3 S08004 [INSPIRE].

[62] CMS collaboration, Charged-particle nuclear modification factors in $\mathrm{PbPb}$ and $p \mathrm{~Pb}$ collisions at $\sqrt{s_{N N}}=5.02 \mathrm{TeV}$, JHEP 04 (2017) 039 [arXiv:1611.01664] [INSPIRE].

[63] CMS collaboration, Transverse momentum and pseudorapidity distributions of charged hadrons in pp collisions at $\sqrt{s}=0.9$ and $2.36 \mathrm{TeV}$, JHEP 02 (2010) 041 [arXiv:1002.0621] [INSPIRE].

[64] T. Sjöstrand et al., An introduction to PYTHIA 8.2, Comput. Phys. Commun. 191 (2015) 159 [arXiv: 1410.3012] [INSPIRE].

[65] CMS collaboration, Event generator tunes obtained from underlying event and multiparton scattering measurements, Eur. Phys. J. C 76 (2016) 155 [arXiv:1512.00815] [InSPIRE].

[66] T. Sjöstrand, S. Mrenna and P.Z. Skands, PYTHIA 6.4 physics and manual, JHEP 05 (2006) 026 [hep-ph/0603175] [INSPIRE].

[67] CMS collaboration, Study of the underlying event at forward rapidity in pp collisions at $\sqrt{s}=0.9,2.76$, and $7 \mathrm{TeV}, \mathrm{JHEP} 04$ (2013) 072 [arXiv: 1302.2394] [INSPIRE]. 
[68] J. Pumplin, D.R. Stump, J. Huston, H.L. Lai, P.M. Nadolsky and W.K. Tung, New generation of parton distributions with uncertainties from global QCD analysis, JHEP $\mathbf{0 7}$ (2002) 012 [hep-ph/0201195] [INSPIRE].

[69] I.P. Lokhtin and A.M. Snigirev, A model of jet quenching in ultrarelativistic heavy ion collisions and high- $p_{\mathrm{T}}$ hadron spectra at RHIC, Eur. Phys. J. C 45 (2006) 211 [hep-ph/0506189] [INSPIRE].

[70] GEANT4 collaboration, GEANT4 - a simulation toolkit, Nucl. Instrum. Meth. A 506 (2003) 250 [INSPIRE].

[71] M. Cacciari, G.P. Salam and G. Soyez, FastJet user manual, Eur. Phys. J. C 72 (2012) 1896 [arXiv:1111.6097] [INSPIRE].

[72] O. Kodolova, I. Vardanyan, A. Nikitenko and A. Oulianov, The performance of the jet identification and reconstruction in heavy ions collisions with CMS detector, Eur. Phys. J. C 50 (2007) 117 [INSPIRE].

[73] CMS collaboration, Jet momentum dependence of jet quenching in $\mathrm{PbPb}$ collisions at $\sqrt{s_{N N}}=2.76 \mathrm{TeV}$, Phys. Lett. B 712 (2012) 176 [arXiv:1202.5022] [INSPIRE].

[74] CMS collaboration, Measurement of the elliptic anisotropy of charged particles produced in $\mathrm{PbPb}$ collisions at $\sqrt{s_{N N}}=2.76 \mathrm{TeV}$, Phys. Rev. C 87 (2013) 014902 [arXiv:1204.1409] [INSPIRE].

[75] CMS collaboration, Study of jet quenching with $Z+$ jet correlations in $\mathrm{Pb}-\mathrm{Pb}$ and pp collisions at $\sqrt{s_{N N}}=5.02 \mathrm{TeV}$, Phys. Rev. Lett. 119 (2017) 082301 [arXiv:1702.01060] [INSPIRE].

[76] P. Berta, M. Spousta, D.W. Miller and R. Leitner, Particle-level pileup subtraction for jets and jet shapes, JHEP 06 (2014) 092 [arXiv:1403.3108] [INSPIRE].

[77] CMS collaboration, Jet energy scale and resolution in the CMS experiment in pp collisions at $8 \mathrm{TeV}, 2017$ JINST $12 \mathrm{P} 02014$ [arXiv: 1607. 03663] [INSPIRE].

[78] G. D'Agostini, A multidimensional unfolding method based on Bayes' theorem, Nucl. Instrum. Meth. A 362 (1995) 487 [INSPIRE].

[79] T. Adye, Unfolding algorithms and tests using RooUnfold, in PHYSTAT 2011, CERN-2011-006.313, CERN, Geneva, Switzerland (2011) [arXiv:1105.1160] [INSPIRE].

[80] A. Höcker and V. Kartvelishvili, SVD approach to data unfolding, Nucl. Instrum. Meth. A 372 (1996) 469 [hep-ph/9509307] [INSPIRE].

[81] CMS collaboration, CMS luminosity calibration for the pp reference run at $\sqrt{s}=5.02 \mathrm{TeV}$, Tech. Rep. CMS-PAS-LUM-16-001, CERN, Geneva, Switzerland (2016).

[82] K.C. Zapp, JEWEL 2.0.0: directions for use, Eur. Phys. J. C 74 (2014) 2762 [arXiv: 1311.0048] [INSPIRE].

[83] Y. He, S. Cao, W. Chen, T. Luo, L.-G. Pang and X.-N. Wang, Interplaying mechanisms behind single inclusive jet suppression in heavy-ion collisions, Phys. Rev. C 99 (2019) 054911 [arXiv: 1809.02525] [INSPIRE].

[84] N.-B. Chang and G.-Y. Qin, Full jet evolution in quark-gluon plasma and nuclear modification of jet production and jet shape in $\mathrm{Pb}+\mathrm{Pb}$ collisions at $2.76 \mathrm{~A} \mathrm{TeV}$ at the CERN Large Hadron Collider, Phys. Rev. C 94 (2016) 024902 [arXiv:1603.01920] [INSPIRE]. 
[85] N.-B. Chang, Y. Tachibana and G.-Y. Qin, Nuclear modification of jet shape for inclusive jets and $\gamma$-jets at the LHC energies, Phys. Lett. B 801 (2020) 135181 [arXiv:1906.09562] [INSPIRE].

[86] B. Schenke, C. Gale and S. Jeon, MARTINI: an event generator for relativistic heavy-ion collisions, Phys. Rev. C 80 (2009) 054913 [arXiv:0909.2037] [INSPIRE].

[87] G.-Y. Qin, J. Ruppert, C. Gale, S. Jeon, G.D. Moore and M.G. Mustafa, Radiative and collisional jet energy loss in the quark-gluon plasma at RHIC, Phys. Rev. Lett. 100 (2008) 072301 [arXiv: 0710.0605] [INSPIRE].

[88] P.B. Arnold, G.D. Moore and L.G. Yaffe, Photon emission from quark gluon plasma: complete leading order results, JHEP 12 (2001) 009 [hep-ph/0111107] [INSPIRE].

[89] P.B. Arnold, G.D. Moore and L.G. Yaffe, Photon emission from ultrarelativistic plasmas, JHEP 11 (2001) 057 [hep-ph/0109064] [INSPIRE].

[90] P.B. Arnold, G.D. Moore and L.G. Yaffe, Photon and gluon emission in relativistic plasmas, JHEP 06 (2002) 030 [hep-ph/0204343] [INSPIRE].

[91] B. Schenke, C. Gale and G.-Y. Qin, The evolving distribution of hard partons traversing a hot strongly interacting plasma, Phys. Rev. C 79 (2009) 054908 [arXiv:0901.3498] [InSPIRE].

[92] J.-W. Qiu, F. Ringer, N. Sato and P. Zurita, Factorization of jet cross sections in heavy-ion collisions, Phys. Rev. Lett. 122 (2019) 252301 [arXiv:1903.01993] [INSPIRE].

[93] R. Baier, Y.L. Dokshitzer, S. Peigne and D. Schiff, Induced gluon radiation in a $Q C D$ medium, Phys. Lett. B 345 (1995) 277 [hep-ph/9411409] [INSPIRE].

[94] J. Casalderrey-Solana, Y. Mehtar-Tani, C.A. Salgado and K. Tywoniuk, New picture of jet quenching dictated by color coherence, Phys. Lett. B 725 (2013) 357 [arXiv:1210.7765] [INSPIRE].

[95] Y. Mehtar-Tani and K. Tywoniuk, Groomed jets in heavy-ion collisions: sensitivity to medium-induced bremsstrahlung, JHEP 04 (2017) 125 [arXiv:1610.08930] [INSPIRE].

[96] Y. Mehtar-Tani and K. Tywoniuk, Sudakov suppression of jets in QCD media, Phys. Rev. D 98 (2018) 051501 [arXiv: 1707.07361] [INSPIRE].

[97] H.T. Li and I. Vitev, Inclusive heavy flavor jet production with semi-inclusive jet functions: from proton to heavy-ion collisions, JHEP 07 (2019) 148 [arXiv:1811.07905] [INSPIRE].

[98] M.D. Sievert, I. Vitev and B. Yoon, A complete set of in-medium splitting functions to any order in opacity, Phys. Lett. B 795 (2019) 502 [arXiv:1903.06170] [INSPIRE]. 


\section{The CMS collaboration}

Yerevan Physics Institute, Yerevan, Armenia

A.M. Sirunyan ${ }^{\dagger}$, A. Tumasyan

\section{Institut für Hochenergiephysik, Wien, Austria}

W. Adam, F. Ambrogi, T. Bergauer, M. Dragicevic, J. Erö, A. Escalante Del Valle, M. Flechl, R. Frühwirth ${ }^{1}$, M. Jeitler ${ }^{1}$, N. Krammer, I. Krätschmer, D. Liko, T. Madlener, I. Mikulec, N. Rad, J. Schieck ${ }^{1}$, R. Schöfbeck, M. Spanring, W. Waltenberger, C.-E. Wulz ${ }^{1}$, M. Zarucki

\section{Institute for Nuclear Problems, Minsk, Belarus}

V. Drugakov, V. Mossolov, J. Suarez Gonzalez

\section{Universiteit Antwerpen, Antwerpen, Belgium}

M.R. Darwish, E.A. De Wolf, D. Di Croce, X. Janssen, T. Kello², A. Lelek, M. Pieters, H. Rejeb Sfar, H. Van Haevermaet, P. Van Mechelen, S. Van Putte, N. Van Remortel

\section{Vrije Universiteit Brussel, Brussel, Belgium}

F. Blekman, E.S. Bols, S.S. Chhibra, J. D'Hondt, J. De Clercq, D. Lontkovskyi, S. Lowette, I. Marchesini, S. Moortgat, Q. Python, S. Tavernier, W. Van Doninck, P. Van Mulders

Université Libre de Bruxelles, Bruxelles, Belgium

D. Beghin, B. Bilin, B. Clerbaux, G. De Lentdecker, H. Delannoy, B. Dorney, L. Favart, A. Grebenyuk, A.K. Kalsi, L. Moureaux, A. Popov, N. Postiau, E. Starling, L. Thomas, C. Vander Velde, P. Vanlaer, D. Vannerom

\section{Ghent University, Ghent, Belgium}

T. Cornelis, D. Dobur, I. Khvastunov ${ }^{3}$, M. Niedziela, C. Roskas, K. Skovpen, M. Tytgat, W. Verbeke, B. Vermassen, M. Vit

\section{Université Catholique de Louvain, Louvain-la-Neuve, Belgium}

G. Bruno, C. Caputo, P. David, C. Delaere, M. Delcourt, A. Giammanco, V. Lemaitre, J. Prisciandaro, A. Saggio, P. Vischia, J. Zobec

\section{Centro Brasileiro de Pesquisas Fisicas, Rio de Janeiro, Brazil}

G.A. Alves, G. Correia Silva, C. Hensel, A. Moraes

Universidade do Estado do Rio de Janeiro, Rio de Janeiro, Brazil

E. Belchior Batista Das Chagas, W. Carvalho, J. Chinellato ${ }^{4}$, E. Coelho, E.M. Da Costa, G.G. Da Silveira ${ }^{5}$, D. De Jesus Damiao, C. De Oliveira Martins, S. Fonseca De Souza, H. Malbouisson, J. Martins ${ }^{6}$, D. Matos Figueiredo, M. Medina Jaime ${ }^{7}$, M. Melo De Almeida, C. Mora Herrera, L. Mundim, H. Nogima, W.L. Prado Da Silva, P. Rebello Teles, L.J. Sanchez Rosas, A. Santoro, A. Sznajder, M. Thiel, E.J. Tonelli Manganote ${ }^{4}$, F. Torres Da Silva De Araujo, A. Vilela Pereira 
Universidade Estadual Paulista ${ }^{a}$, Universidade Federal do $\mathrm{ABC}^{b}$, São Paulo, Brazil

C.A. Bernardes ${ }^{a}$, L. Calligaris ${ }^{a}$, T.R. Fernandez Perez Tomei ${ }^{a}$, E.M. Gregores ${ }^{b}$, D.S. Lemos ${ }^{a}$, P.G. Mercadante ${ }^{b}$, S.F. Novaes ${ }^{a}$, Sandra S. Padula ${ }^{a}$

Institute for Nuclear Research and Nuclear Energy, Bulgarian Academy of Sciences, Sofia, Bulgaria

A. Aleksandrov, G. Antchev, R. Hadjiiska, P. Iaydjiev, M. Misheva, M. Rodozov, M. Shopova, G. Sultanov

University of Sofia, Sofia, Bulgaria

M. Bonchev, A. Dimitrov, T. Ivanov, L. Litov, B. Pavlov, P. Petkov, A. Petrov

Beihang University, Beijing, China

W. Fang $^{2}$, X. Gao ${ }^{2}$, L. Yuan

Department of Physics, Tsinghua University, Beijing, China

M. Ahmad, Z. Hu, Y. Wang

Institute of High Energy Physics, Beijing, China

G.M. Chen ${ }^{8}$, H.S. Chen ${ }^{8}$, M. Chen, C.H. Jiang, D. Leggat, H. Liao, Z. Liu, A. Spiezia, J. Tao, E. Yazgan, H. Zhang, S. Zhang ${ }^{8}$, J. Zhao

State Key Laboratory of Nuclear Physics and Technology, Peking University, Beijing, China

A. Agapitos, Y. Ban, G. Chen, A. Levin, J. Li, L. Li, Q. Li, Y. Mao, S.J. Qian, D. Wang, Q. Wang

Zhejiang University, Hangzhou, China

M. Xiao

Universidad de Los Andes, Bogota, Colombia

C. Avila, A. Cabrera, C. Florez, C.F. González Hernández, M.A. Segura Delgado

Universidad de Antioquia, Medellin, Colombia

J. Mejia Guisao, J.D. Ruiz Alvarez, C.A. Salazar González, N. Vanegas Arbelaez

University of Split, Faculty of Electrical Engineering, Mechanical Engineering and Naval Architecture, Split, Croatia

D. Giljanović, N. Godinovic, D. Lelas, I. Puljak, T. Sculac

University of Split, Faculty of Science, Split, Croatia

Z. Antunovic, M. Kovac

Institute Rudjer Boskovic, Zagreb, Croatia

V. Brigljevic, D. Ferencek, K. Kadija, B. Mesic, M. Roguljic, A. Starodumov ${ }^{9}$, T. Susa

University of Cyprus, Nicosia, Cyprus

M.W. Ather, A. Attikis, E. Erodotou, A. Ioannou, M. Kolosova, S. Konstantinou,

G. Mavromanolakis, J. Mousa, C. Nicolaou, F. Ptochos, P.A. Razis, H. Rykaczewski,

H. Saka, D. Tsiakkouri 
Charles University, Prague, Czech Republic

M. Finger ${ }^{10}$, M. Finger Jr. ${ }^{10}$, A. Kveton, J. Tomsa

Escuela Politecnica Nacional, Quito, Ecuador

E. Ayala

Universidad San Francisco de Quito, Quito, Ecuador

E. Carrera Jarrin

Academy of Scientific Research and Technology of the Arab Republic of Egypt, Egyptian Network of High Energy Physics, Cairo, Egypt

M.A. Mahmoud ${ }^{11,12}$, Y. Mohammed ${ }^{11}$

National Institute of Chemical Physics and Biophysics, Tallinn, Estonia

S. Bhowmik, A. Carvalho Antunes De Oliveira, R.K. Dewanjee, K. Ehataht, M. Kadastik, M. Raidal, C. Veelken

Department of Physics, University of Helsinki, Helsinki, Finland

P. Eerola, L. Forthomme, H. Kirschenmann, K. Osterberg, M. Voutilainen

Helsinki Institute of Physics, Helsinki, Finland

F. Garcia, J. Havukainen, J.K. Heikkilä, V. Karimäki, M.S. Kim, R. Kinnunen, T. Lampén, K. Lassila-Perini, S. Laurila, S. Lehti, T. Lindén, H. Siikonen, E. Tuominen, J. Tuominiemi

Lappeenranta University of Technology, Lappeenranta, Finland

P. Luukka, T. Tuuva

IRFU, CEA, Université Paris-Saclay, Gif-sur-Yvette, France

M. Besancon, F. Couderc, M. Dejardin, D. Denegri, B. Fabbro, J.L. Faure, F. Ferri,

S. Ganjour, A. Givernaud, P. Gras, G. Hamel de Monchenault, P. Jarry, C. Leloup,

B. Lenzi, E. Locci, J. Malcles, J. Rander, A. Rosowsky, M.Ö. Sahin, A. Savoy-Navarro ${ }^{13}$,

M. Titov, G.B. Yu

Laboratoire Leprince-Ringuet, CNRS/IN2P3, Ecole Polytechnique, Institut Polytechnique de Paris, Palaiseau, France

S. Ahuja, C. Amendola, F. Beaudette, M. Bonanomi, P. Busson, C. Charlot, B. Diab, G. Falmagne, R. Granier de Cassagnac, I. Kucher, A. Lobanov, C. Martin Perez, M. Nguyen, C. Ochando, P. Paganini, J. Rembser, R. Salerno, J.B. Sauvan, Y. Sirois, A. Zabi, A. Zghiche

Université de Strasbourg, CNRS, IPHC UMR 7178, Strasbourg, France J.-L. Agram ${ }^{14}$, J. Andrea, D. Bloch, G. Bourgatte, J.-M. Brom, E.C. Chabert, C. Collard, E. Conte ${ }^{14}$, J.-C. Fontaine ${ }^{14}$, D. Gelé, U. Goerlach, C. Grimault, A.-C. Le Bihan, N. Tonon, P. Van Hove

Centre de Calcul de l'Institut National de Physique Nucleaire et de Physique des Particules, CNRS/IN2P3, Villeurbanne, France

S. Gadrat 
Université de Lyon, Université Claude Bernard Lyon 1, CNRS-IN2P3, Institut de Physique Nucléaire de Lyon, Villeurbanne, France

S. Beauceron, C. Bernet, G. Boudoul, C. Camen, A. Carle, N. Chanon, R. Chierici, D. Contardo, P. Depasse, H. El Mamouni, J. Fay, S. Gascon, M. Gouzevitch, B. Ille, Sa. Jain, I.B. Laktineh, H. Lattaud, A. Lesauvage, M. Lethuillier, L. Mirabito, S. Perries, V. Sordini, L. Torterotot, G. Touquet, M. Vander Donckt, S. Viret

Georgian Technical University, Tbilisi, Georgia

G. Adamov

Tbilisi State University, Tbilisi, Georgia

I. Bagaturia ${ }^{15}$

RWTH Aachen University, I. Physikalisches Institut, Aachen, Germany

C. Autermann, L. Feld, K. Klein, M. Lipinski, D. Meuser, A. Pauls, M. Preuten, M.P. Rauch, J. Schulz, M. Teroerde

RWTH Aachen University, III. Physikalisches Institut A, Aachen, Germany

M. Erdmann, B. Fischer, S. Ghosh, T. Hebbeker, K. Hoepfner, H. Keller, L. Mastrolorenzo, M. Merschmeyer, A. Meyer, P. Millet, G. Mocellin, S. Mondal, S. Mukherjee, D. Noll, A. Novak, T. Pook, A. Pozdnyakov, T. Quast, M. Radziej, Y. Rath, H. Reithler, J. Roemer, A. Schmidt, S.C. Schuler, A. Sharma, S. Wiedenbeck, S. Zaleski

RWTH Aachen University, III. Physikalisches Institut B, Aachen, Germany G. Flügge, W. Haj Ahmad ${ }^{16}$, O. Hlushchenko, T. Kress, T. Müller, A. Nowack, C. Pistone, O. Pooth, D. Roy, H. Sert, A. Stahl ${ }^{17}$

\section{Deutsches Elektronen-Synchrotron, Hamburg, Germany}

M. Aldaya Martin, P. Asmuss, I. Babounikau, H. Bakhshiansohi, K. Beernaert, O. Behnke, A. Bermúdez Martínez, A.A. Bin Anuar, K. Borras ${ }^{18}$, V. Botta, A. Campbell, A. Cardini, P. Connor, S. Consuegra Rodríguez, C. Contreras-Campana, V. Danilov, A. De Wit, M.M. Defranchis, C. Diez Pardos, D. Domínguez Damiani, G. Eckerlin, D. Eckstein, T. Eichhorn, A. Elwood, E. Eren, E. Gallo ${ }^{19}$, A. Geiser, A. Grohsjean, M. Guthoff, M. Haranko, A. Harb, A. Jafari, N.Z. Jomhari, H. Jung, A. Kasem ${ }^{18}$, M. Kasemann, H. Kaveh, J. Keaveney, C. Kleinwort, J. Knolle, D. Krücker, W. Lange, T. Lenz, J. Lidrych, K. Lipka, W. Lohmann ${ }^{20}$, R. Mankel, I.-A. Melzer-Pellmann, A.B. Meyer, M. Meyer, M. Missiroli, J. Mnich, A. Mussgiller, V. Myronenko, D. Pérez Adán, S.K. Pflitsch, D. Pitzl, A. Raspereza, A. Saibel, M. Savitskyi, V. Scheurer, P. Schütze, C. Schwanenberger, R. Shevchenko, A. Singh, R.E. Sosa Ricardo, H. Tholen, O. Turkot, A. Vagnerini, M. Van De Klundert, R. Walsh, Y. Wen, K. Wichmann, C. Wissing, O. Zenaiev, R. Zlebcik

\section{University of Hamburg, Hamburg, Germany}

R. Aggleton, S. Bein, L. Benato, A. Benecke, T. Dreyer, A. Ebrahimi, F. Feindt, A. Fröhlich, C. Garbers, E. Garutti, D. Gonzalez, P. Gunnellini, J. Haller, A. Hinzmann, A. Karavdina, G. Kasieczka, R. Klanner, R. Kogler, N. Kovalchuk, S. Kurz, V. Kutzner, J. Lange, T. Lange, A. Malara, J. Multhaup, C.E.N. Niemeyer, A. Reimers, O. Rieger, P. Schleper, S. Schumann, J. Schwandt, J. Sonneveld, H. Stadie, G. Steinbrück, B. Vormwald, I. Zoi 
Karlsruher Institut fuer Technologie, Karlsruhe, Germany

M. Akbiyik, M. Baselga, S. Baur, T. Berger, E. Butz, R. Caspart, T. Chwalek, W. De Boer, A. Dierlamm, K. El Morabit, N. Faltermann, M. Giffels, A. Gottmann, F. Hartmann ${ }^{17}$, C. Heidecker, U. Husemann, M.A. Iqbal, S. Kudella, S. Maier, S. Mitra, M.U. Mozer, D. Müller, Th. Müller, M. Musich, A. Nürnberg, G. Quast, K. Rabbertz, D. Savoiu, D. Schäfer, M. Schnepf, M. Schröder, I. Shvetsov, H.J. Simonis, R. Ulrich, M. Wassmer, M. Weber, C. Wöhrmann, R. Wolf, S. Wozniewski

Institute of Nuclear and Particle Physics (INPP), NCSR Demokritos, Aghia Paraskevi, Greece

G. Anagnostou, P. Asenov, G. Daskalakis, T. Geralis, A. Kyriakis, D. Loukas, G. Paspalaki, A. Stakia

National and Kapodistrian University of Athens, Athens, Greece

M. Diamantopoulou, G. Karathanasis, P. Kontaxakis, A. Manousakis-Katsikakis, A. Panagiotou, I. Papavergou, N. Saoulidou, K. Theofilatos, K. Vellidis, E. Vourliotis

National Technical University of Athens, Athens, Greece

G. Bakas, K. Kousouris, I. Papakrivopoulos, G. Tsipolitis, A. Zacharopoulou

University of Ioánnina, Ioánnina, Greece

I. Evangelou, C. Foudas, P. Gianneios, P. Katsoulis, P. Kokkas, S. Mallios, K. Manitara, N. Manthos, I. Papadopoulos, J. Strologas, F.A. Triantis, D. Tsitsonis

MTA-ELTE Lendület CMS Particle and Nuclear Physics Group, Eötvös Loránd University, Budapest, Hungary

M. Bartók ${ }^{21}$, R. Chudasama, M. Csanad, P. Major, K. Mandal, A. Mehta, G. Pasztor, O. Surányi, G.I. Veres

Wigner Research Centre for Physics, Budapest, Hungary

G. Bencze, C. Hajdu, D. Horvath ${ }^{22}$, F. Sikler, V. Veszpremi, G. Vesztergombi ${ }^{\dagger}$

Institute of Nuclear Research ATOMKI, Debrecen, Hungary

N. Beni, S. Czellar, J. Karancsi ${ }^{21}$, J. Molnar, Z. Szillasi

Institute of Physics, University of Debrecen, Debrecen, Hungary

P. Raics, D. Teyssier, Z.L. Trocsanyi, B. Ujvari

Eszterhazy Karoly University, Karoly Robert Campus, Gyongyos, Hungary

T. Csorgo, W.J. Metzger, F. Nemes, T. Novak

Indian Institute of Science (IISc), Bangalore, India

S. Choudhury, J.R. Komaragiri, P.C. Tiwari

National Institute of Science Education and Research, HBNI, Bhubaneswar, India

S. Bahinipati ${ }^{24}$, C. Kar, G. Kole, P. Mal, V.K. Muraleedharan Nair Bindhu, A. Nayak ${ }^{25}$, D.K. Sahoo ${ }^{24}$, S.K. Swain 
Panjab University, Chandigarh, India

S. Bansal, S.B. Beri, V. Bhatnagar, S. Chauhan, N. Dhingra ${ }^{26}$, R. Gupta, A. Kaur, M. Kaur, S. Kaur, P. Kumari, M. Lohan, M. Meena, K. Sandeep, S. Sharma, J.B. Singh, A.K. Virdi

University of Delhi, Delhi, India

A. Bhardwaj, B.C. Choudhary, R.B. Garg, M. Gola, S. Keshri, Ashok Kumar, M. Naimuddin, P. Priyanka, K. Ranjan, Aashaq Shah, R. Sharma

Saha Institute of Nuclear Physics, HBNI, Kolkata, India

R. Bhardwaj ${ }^{27}$, M. Bharti ${ }^{27}$, R. Bhattacharya, S. Bhattacharya, U. Bhawandeep ${ }^{27}$,

D. Bhowmik, S. Dutta, S. Ghosh, B. Gomber ${ }^{28}$, M. Maity ${ }^{29}$, K. Mondal, S. Nandan,

A. Purohit, P.K. Rout, G. Saha, S. Sarkar, M. Sharan, B. Singh ${ }^{27}$, S. Thakur ${ }^{27}$

Indian Institute of Technology Madras, Madras, India

P.K. Behera, S.C. Behera, P. Kalbhor, A. Muhammad, P.R. Pujahari, A. Sharma, A.K. Sikdar

Bhabha Atomic Research Centre, Mumbai, India

D. Dutta, V. Jha, D.K. Mishra, P.K. Netrakanti, L.M. Pant, P. Shukla

Tata Institute of Fundamental Research-A, Mumbai, India

T. Aziz, M.A. Bhat, S. Dugad, G.B. Mohanty, N. Sur, Ravindra Kumar Verma

Tata Institute of Fundamental Research-B, Mumbai, India

S. Banerjee, S. Bhattacharya, S. Chatterjee, P. Das, M. Guchait, S. Karmakar, S. Kumar,

G. Majumder, K. Mazumdar, N. Sahoo, S. Sawant

Indian Institute of Science Education and Research (IISER), Pune, India

S. Dube, B. Kansal, A. Kapoor, K. Kothekar, S. Pandey, A. Rane, A. Rastogi, S. Sharma

Institute for Research in Fundamental Sciences (IPM), Tehran, Iran

S. Chenarani, S.M. Etesami, M. Khakzad, M. Mohammadi Najafabadi, M. Naseri, F. Rezaei Hosseinabadi

University College Dublin, Dublin, Ireland

M. Felcini, M. Grunewald

INFN Sezione di Bari ${ }^{a}$, Università di Bari ${ }^{b}$, Politecnico di Bari ${ }^{c}$, Bari, Italy M. Abbrescia ${ }^{a, b}$, R. Aly ${ }^{a, b, 30}$, C. Calabria ${ }^{a, b}$, A. Colaleo ${ }^{a}$, D. Creanza ${ }^{a, c}$, L. Cristella $^{a, b}$, N. De Filippis ${ }^{a, c}$, M. De Palma ${ }^{a, b}$, A. Di Florio ${ }^{a, b}$, W. Elmetenawee ${ }^{a, b}$ L. Fiore $^{a}$,

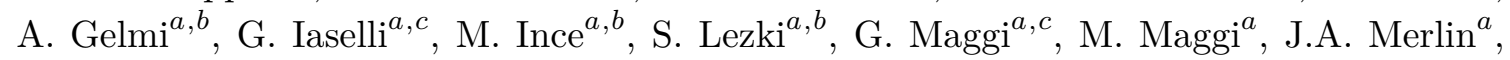
G. Miniello ${ }^{a, b}$, S. My ${ }^{a, b}$, S. Nuzzo ${ }^{a, b}$, A. Pompili ${ }^{a, b}$, G. Pugliese ${ }^{a, c}$, R. Radogna ${ }^{a}$,

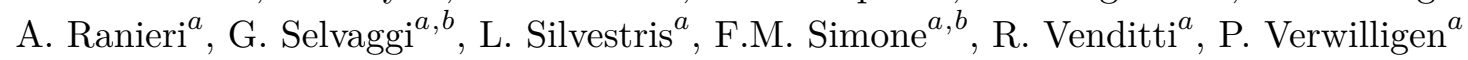

INFN Sezione di Bologna $^{a}$, Università di Bologna ${ }^{b}$, Bologna, Italy

G. Abbiendi ${ }^{a}$, C. Battilana ${ }^{a, b}$, D. Bonacorsi ${ }^{a, b}$, L. Borgonovi $^{a, b}$, S. Braibant-Giacomelli $^{a, b}$, R. Campanini ${ }^{a, b}$, P. Capiluppi ${ }^{a, b}$, A. Castro ${ }^{a, b}$, F.R. Cavallo $^{a}$, C. Ciocca $^{a}$, G. Codispoti $^{a, b}{ }^{,}$ M. Cuffiani ${ }^{a, b}$, G.M. Dallavalle ${ }^{a}$, F. Fabbri ${ }^{a}$, A. Fanfani ${ }^{a, b}$, E. Fontanesi $^{a, b}$, P. Giacomelli $^{a}$, 
L. Giommi ${ }^{a, b}$, C. Grandi ${ }^{a}$, L. Guiducci ${ }^{a, b}$, F. Iemmi ${ }^{a, b}$, S. Lo Meo ${ }^{a, 31}$, S. Marcellini ${ }^{a}$,

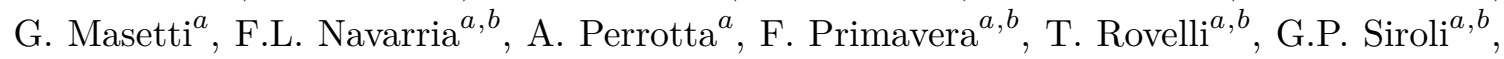
N. $\operatorname{Tosi}^{a}$

INFN Sezione di Catania ${ }^{a}$, Università di Catania ${ }^{b}$, Catania, Italy

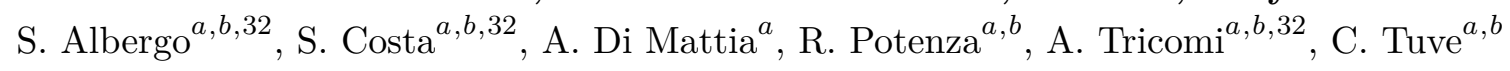
INFN Sezione di Firenze ${ }^{a}$, Università di Firenze ${ }^{b}$, Firenze, Italy

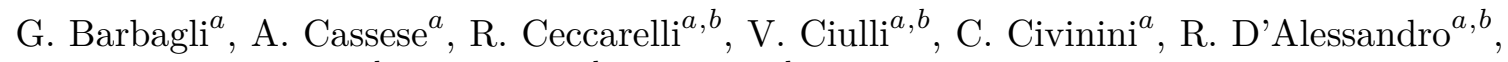
F. Fiori ${ }^{a}$, E. Focardi ${ }^{a, b}$, G. Latino $^{a, b}$, P. Lenzi $^{a, b}$, M. Meschini $^{a}$, S. Paoletti $^{a}$, G. Sguazzoni $^{a}$, L. Viliani ${ }^{a}$

\section{INFN Laboratori Nazionali di Frascati, Frascati, Italy}

L. Benussi, S. Bianco, D. Piccolo

INFN Sezione di Genova ${ }^{a}$, Università di Genova ${ }^{b}$, Genova, Italy

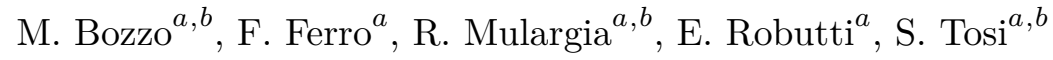

INFN Sezione di Milano-Bicocca ${ }^{a}$, Università di Milano-Bicocca ${ }^{b}$, Milano, Italy

A. Benaglia ${ }^{a}$, A. Beschi ${ }^{a, b}$, F. Brivio ${ }^{a, b}$, V. Ciriolo ${ }^{a, b, 17}$, M.E. Dinardo ${ }^{a, b}$, P. Dini ${ }^{a}$,

S. Gennai ${ }^{a}$, A. Ghezzi ${ }^{a, b}$, P. Govoni ${ }^{a, b}$, L. Guzzi ${ }^{a, b}$, M. Malberti ${ }^{a}$, S. Malvezzi ${ }^{a}$,

D. Menasce ${ }^{a}$, F. Monti ${ }^{a, b}$, L. Moroni ${ }^{a}$, M. Paganoni ${ }^{a, b}$, D. Pedrini ${ }^{a}$, S. Ragazzi ${ }^{a, b}$,

T. Tabarelli de Fatis ${ }^{a, b}$, D. Valsecchi ${ }^{a, b, 17}$, D. Zuolo ${ }^{a, b}$

INFN Sezione di Napoli ${ }^{a}$, Università di Napoli 'Federico II' ${ }^{b}$, Napoli, Italy, Università della Basilicata ${ }^{c}$, Potenza, Italy, Università G. Marconi ${ }^{d}$, Roma, Italy

S. Buontempo ${ }^{a}$, N. Cavallo ${ }^{a, c}$, A. De Iorio ${ }^{a, b}$, A. Di Crescenzo ${ }^{a, b}$, F. Fabozzi $^{a, c}$, F. Fienga $^{a}$,

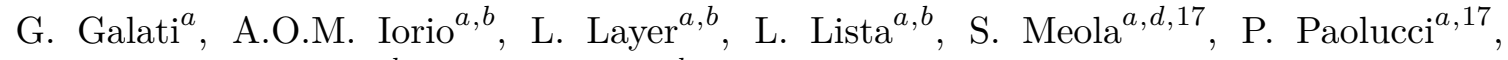
B. Rossi ${ }^{a}$, C. Sciacca ${ }^{a, b}$, E. Voevodina ${ }^{a, b}$

INFN Sezione di Padova ${ }^{a}$, Università di Padova ${ }^{b}$, Padova, Italy, Università di Trento ${ }^{c}$, Trento, Italy

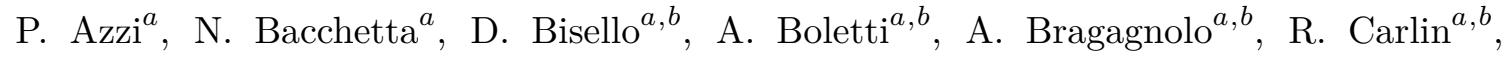

P. Checchia ${ }^{a}$, P. De Castro Manzano $^{a}$, T. Dorigo ${ }^{a}$, U. Dosselli ${ }^{a}$, F. Gasparini ${ }^{a, b}$,

U. Gasparini ${ }^{a, b}$, A. Gozzelino ${ }^{a}$, S.Y. $\operatorname{Hoh}^{a, b}$, M. Margoni ${ }^{a, b}$, A.T. Meneguzzo ${ }^{a, b}$, J. Pazzini ${ }^{a, b}$, M. Presilla ${ }^{b}$, P. Ronchese ${ }^{a, b}$, R. Rossin ${ }^{a, b}$, F. Simonetto ${ }^{a, b}$, A. Tiko ${ }^{a}$, M. Tosi ${ }^{a, b}$, M. Zanetti ${ }^{a, b}$, P. Zotto ${ }^{a, b}$, A. Zucchetta ${ }^{a, b}$, G. Zumerle ${ }^{a, b}$

INFN Sezione di Pavia ${ }^{a}$, Università di Pavia ${ }^{b}$, Pavia, Italy

A. Braghieri ${ }^{a}$, D. Fiorina ${ }^{a, b}$, P. Montagna ${ }^{a, b}$, S.P. Ratti ${ }^{a, b}$, V. $\operatorname{Re}^{a}$, M. Ressegotti $^{a, b}$, C. Riccardi ${ }^{a, b}$, P. Salvini ${ }^{a}$, I. Vai $^{a}$, P. Vitulo ${ }^{a, b}$

INFN Sezione di Perugia ${ }^{a}$, Università di Perugia ${ }^{b}$, Perugia, Italy

M. Biasini ${ }^{a, b}$, G.M. Bilei ${ }^{a}$, D. Ciangottini ${ }^{a, b}$, L. Fanò ${ }^{a, b}$, P. Lariccia ${ }^{a, b}$, R. Leonardi ${ }^{a, b}$,

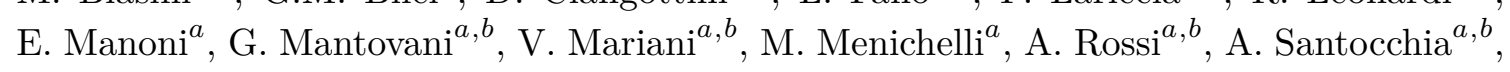
D. Spiga ${ }^{a}$ 
INFN Sezione di Pisa ${ }^{a}$, Università di Pisa ${ }^{b}$, Scuola Normale Superiore di Pisa ${ }^{c}$, Pisa Italy, Università di Siena ${ }^{d}$, Siena, Italy

K. Androsov $^{a}$, P. Azzurri ${ }^{a}$, G. Bagliesi ${ }^{a}$, V. Bertacchi ${ }^{a, c}$, L. Bianchini $^{a}$, T. Boccali ${ }^{a}$, R. Castaldi ${ }^{a}$, M.A. Ciocci ${ }^{a, b}$, R. Dell'Orso ${ }^{a}$, S. Donato ${ }^{a}$, L. Giannini $^{a, c}$, A. Giassi $^{a}$, M.T. Grippo ${ }^{a}$, F. Ligabue ${ }^{a, c}$, E. Manca ${ }^{a, c}$, G. Mandorli ${ }^{a, c}$, A. Messineo ${ }^{a, b}$, F. Palla ${ }^{a}$, A. Rizzi ${ }^{a, b}$, G. Rolandi ${ }^{a, c}$, S. Roy Chowdhury $^{a, c}$, A. Scribano $^{a}$, P. Spagnolo $^{a}$, R. Tenchini $^{a}$, G. Tonelli ${ }^{a, b}$, N. Turini ${ }^{a, d}$, A. Venturi ${ }^{a}$, P.G. Verdini ${ }^{a}$

INFN Sezione di Roma ${ }^{a}$, Sapienza Università di Roma ${ }^{b}$, Rome, Italy

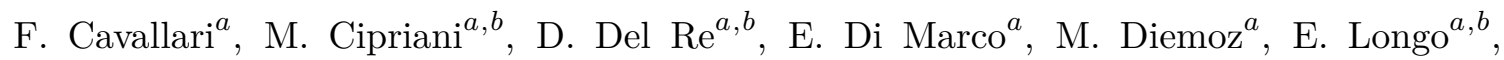
P. Meridiani ${ }^{a}$, G. Organtini ${ }^{a, b}$, F. Pandolfi ${ }^{a}$, R. Paramatti ${ }^{a, b}$, C. Quaranta ${ }^{a, b}$,

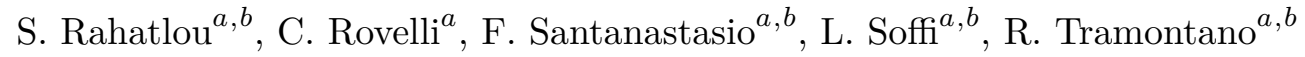

INFN Sezione di Torino ${ }^{a}$, Università di Torino ${ }^{b}$, Torino, Italy, Università del Piemonte Orientale ${ }^{c}$, Novara, Italy

N. Amapane ${ }^{a, b}$, R. Arcidiacono ${ }^{a, c}$, S. Argiro ${ }^{a, b}$, M. Arneodo ${ }^{a, c}$, N. Bartosik ${ }^{a}$, R. Bellan ${ }^{a, b}$, A. Bellora ${ }^{a, b}$, C. Biino ${ }^{a}$, A. Cappati ${ }^{a, b}$, N. Cartiglia ${ }^{a}$, S. Cometti ${ }^{a}$, M. $\operatorname{Costa}^{a, b}$, R. Covarelli ${ }^{a, b}$, N. Demaria ${ }^{a}$, J.R. González Fernández ${ }^{a}$, B. Kiani ${ }^{a, b}$, F. Legger ${ }^{a}$, C. Mariotti ${ }^{a}$, S. Maselli ${ }^{a}$, E. Migliore ${ }^{a, b}$, V. Monaco ${ }^{a, b}$, E. Monteil ${ }^{a, b}$, M. Monteno ${ }^{a}$, M.M. Obertino ${ }^{a, b}$, G. Ortona ${ }^{a}$, L. Pacher $^{a}, b$, N. Pastrone ${ }^{a}$, M. Pelliccioni ${ }^{a}$, G.L. Pinna Angioni ${ }^{a, b}$, A. Romero ${ }^{a, b}$, M. Ruspa $^{a, c}$, R. Salvatico ${ }^{a, b}$, V. Sola ${ }^{a}$, A. Solano ${ }^{a, b}$, D. Soldi ${ }^{a, b}$, A. Staiano ${ }^{a}$, D. Trocino ${ }^{a, b}$

INFN Sezione di Trieste ${ }^{a}$, Università di Trieste ${ }^{b}$, Trieste, Italy

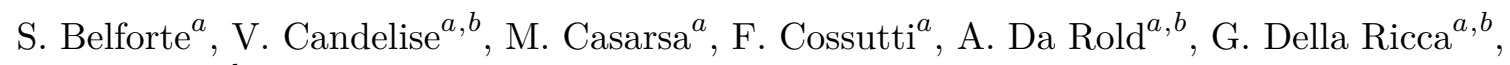
F. Vazzoler ${ }^{a, b}$, A. Zanetti ${ }^{a}$

Kyungpook National University, Daegu, Korea

B. Kim, D.H. Kim, G.N. Kim, J. Lee, S.W. Lee, C.S. Moon, Y.D. Oh, S.I. Pak, S. Sekmen, D.C. Son, Y.C. Yang

Chonnam National University, Institute for Universe and Elementary Particles, Kwangju, Korea

H. Kim, D.H. Moon

Hanyang University, Seoul, Korea

B. Francois, T.J. Kim, J. Park

Korea University, Seoul, Korea

S. Cho, S. Choi, Y. Go, S. Ha, B. Hong, K. Lee, K.S. Lee, J. Lim, J. Park, S.K. Park, Y. Roh, J. Yoo

Kyung Hee University, Department of Physics, Seoul, Republic of Korea

J. Goh

Sejong University, Seoul, Korea

H.S. Kim 
Seoul National University, Seoul, Korea

J. Almond, J.H. Bhyun, J. Choi, S. Jeon, J. Kim, J.S. Kim, H. Lee, K. Lee, S. Lee, K. Nam, M. Oh, S.B. Oh, B.C. Radburn-Smith, U.K. Yang, H.D. Yoo, I. Yoon

University of Seoul, Seoul, Korea

D. Jeon, J.H. Kim, J.S.H. Lee, I.C. Park, I.J. Watson

Sungkyunkwan University, Suwon, Korea

Y. Choi, C. Hwang, Y. Jeong, J. Lee, Y. Lee, I. Yu

Riga Technical University, Riga, Latvia

V. Veckalns ${ }^{33}$

Vilnius University, Vilnius, Lithuania

V. Dudenas, A. Juodagalvis, A. Rinkevicius, G. Tamulaitis, J. Vaitkus

National Centre for Particle Physics, Universiti Malaya, Kuala Lumpur, Malaysia

F. Mohamad Idris ${ }^{34}$, W.A.T. Wan Abdullah, M.N. Yusli, Z. Zolkapli

Universidad de Sonora (UNISON), Hermosillo, Mexico

J.F. Benitez, A. Castaneda Hernandez, J.A. Murillo Quijada, L. Valencia Palomo

Centro de Investigacion y de Estudios Avanzados del IPN, Mexico City, Mexico

H. Castilla-Valdez, E. De La Cruz-Burelo, I. Heredia-De La Cruz ${ }^{35}$, R. Lopez-Fernandez,

A. Sanchez-Hernandez

Universidad Iberoamericana, Mexico City, Mexico

S. Carrillo Moreno, C. Oropeza Barrera, M. Ramirez-Garcia, F. Vazquez Valencia

Benemerita Universidad Autonoma de Puebla, Puebla, Mexico

J. Eysermans, I. Pedraza, H.A. Salazar Ibarguen, C. Uribe Estrada

Universidad Autónoma de San Luis Potosí, San Luis Potosí, Mexico

A. Morelos Pineda

University of Montenegro, Podgorica, Montenegro

J. Mijuskovic ${ }^{3}$, N. Raicevic

University of Auckland, Auckland, New Zealand

D. Krofcheck

University of Canterbury, Christchurch, New Zealand

S. Bheesette, P.H. Butler, P. Lujan

National Centre for Physics, Quaid-I-Azam University, Islamabad, Pakistan

A. Ahmad, M. Ahmad, M.I.M. Awan, Q. Hassan, H.R. Hoorani, W.A. Khan, M.A. Shah, M. Shoaib, M. Waqas

AGH University of Science and Technology Faculty of Computer Science, Electronics and Telecommunications, Krakow, Poland

V. Avati, L. Grzanka, M. Malawski 
National Centre for Nuclear Research, Swierk, Poland

H. Bialkowska, M. Bluj, B. Boimska, M. Górski, M. Kazana, M. Szleper, P. Zalewski

Institute of Experimental Physics, Faculty of Physics, University of Warsaw, Warsaw, Poland

K. Bunkowski, A. Byszuk ${ }^{36}$, K. Doroba, A. Kalinowski, M. Konecki, J. Krolikowski, M. Olszewski, M. Walczak

Laboratório de Instrumentação e Física Experimental de Partículas, Lisboa, Portugal

M. Araujo, P. Bargassa, D. Bastos, A. Di Francesco, P. Faccioli, B. Galinhas, M. Gallinaro,

J. Hollar, N. Leonardo, T. Niknejad, J. Seixas, K. Shchelina, G. Strong, O. Toldaiev,

J. Varela

Joint Institute for Nuclear Research, Dubna, Russia

S. Afanasiev, P. Bunin, Y. Ershov, M. Gavrilenko, A. Golunov, I. Golutvin, N. Gorbounov,

I. Gorbunov, A. Kamenev, V. Karjavine, A. Lanev, A. Malakhov, V. Matveev ${ }^{37,38}$,

P. Moisenz, V. Palichik, V. Perelygin, S. Shmatov, V. Smirnov, A. Zarubin, V. Zhiltsov

Petersburg Nuclear Physics Institute, Gatchina (St. Petersburg), Russia

L. Chtchipounov, V. Golovtcov, Y. Ivanov, V. Kim ${ }^{39}$, E. Kuznetsova ${ }^{40}$, P. Levchenko,

V. Murzin, V. Oreshkin, I. Smirnov, D. Sosnov, V. Sulimov, L. Uvarov, A. Vorobyev

Institute for Nuclear Research, Moscow, Russia

Yu. Andreev, A. Dermenev, S. Gninenko, N. Golubev, A. Karneyeu, M. Kirsanov,

N. Krasnikov, A. Pashenkov, D. Tlisov, A. Toropin

Institute for Theoretical and Experimental Physics named by A.I. Alikhanov of NRC 'Kurchatov Institute', Moscow, Russia

V. Epshteyn, V. Gavrilov, N. Lychkovskaya, A. Nikitenko ${ }^{41}$, V. Popov, I. Pozdnyakov,

G. Safronov, A. Spiridonov, A. Stepennov, M. Toms, E. Vlasov, A. Zhokin

Moscow Institute of Physics and Technology, Moscow, Russia

T. Aushev

National Research Nuclear University 'Moscow Engineering Physics Institute' (MEPhI), Moscow, Russia

M. Chadeeva ${ }^{42}$, P. Parygin, D. Philippov, E. Popova, V. Rusinov

P.N. Lebedev Physical Institute, Moscow, Russia

V. Andreev, M. Azarkin, I. Dremin, M. Kirakosyan, A. Terkulov

Skobeltsyn Institute of Nuclear Physics, Lomonosov Moscow State University, Moscow, Russia

A. Belyaev, E. Boos, A. Ershov, A. Gribushin, A. Kaminskiy ${ }^{43}$, O. Kodolova, V. Korotkikh, I. Lokhtin, S. Obraztsov, S. Petrushanko, V. Savrin, A. Snigirev, I. Vardanyan

Novosibirsk State University (NSU), Novosibirsk, Russia

A. Barnyakov ${ }^{44}$, V. Blinov ${ }^{44}$, T. Dimova ${ }^{44}$, L. Kardapoltsev $^{44}$, Y. Skovpen ${ }^{44}$ 
Institute for High Energy Physics of National Research Centre 'Kurchatov Institute', Protvino, Russia

I. Azhgirey, I. Bayshev, S. Bitioukov, V. Kachanov, D. Konstantinov, P. Mandrik, V. Petrov, R. Ryutin, S. Slabospitskii, A. Sobol, S. Troshin, N. Tyurin, A. Uzunian, A. Volkov

\section{National Research Tomsk Polytechnic University, Tomsk, Russia}

A. Babaev, A. Iuzhakov, V. Okhotnikov

Tomsk State University, Tomsk, Russia

V. Borchsh, V. Ivanchenko, E. Tcherniaev

University of Belgrade: Faculty of Physics and VINCA Institute of Nuclear Sciences, Belgrade, Serbia

P. Adzic ${ }^{45}$, P. Cirkovic, M. Dordevic, P. Milenovic, J. Milosevic, M. Stojanovic

Centro de Investigaciones Energéticas Medioambientales y Tecnológicas (CIEMAT), Madrid, Spain

M. Aguilar-Benitez, J. Alcaraz Maestre, A. Álvarez Fernández, I. Bachiller, M. Barrio Luna, Cristina F. Bedoya, J.A. Brochero Cifuentes, C.A. Carrillo Montoya, M. Cepeda, M. Cerrada, N. Colino, B. De La Cruz, A. Delgado Peris, J.P. Fernández Ramos, J. Flix, M.C. Fouz, O. Gonzalez Lopez, S. Goy Lopez, J.M. Hernandez, M.I. Josa, D. Moran, Á. Navarro Tobar, A. Pérez-Calero Yzquierdo, J. Puerta Pelayo, I. Redondo, L. Romero, S. Sánchez Navas, M.S. Soares, A. Triossi, C. Willmott

Universidad Autónoma de Madrid, Madrid, Spain

C. Albajar, J.F. de Trocóniz, R. Reyes-Almanza

Universidad de Oviedo, Instituto Universitario de Ciencias y Tecnologías Espaciales de Asturias (ICTEA), Oviedo, Spain

B. Alvarez Gonzalez, J. Cuevas, C. Erice, J. Fernandez Menendez, S. Folgueras, I. Gonzalez Caballero, E. Palencia Cortezon, C. Ramón Álvarez, V. Rodríguez Bouza, S. Sanchez Cruz

Instituto de Física de Cantabria (IFCA), CSIC-Universidad de Cantabria, Santander, Spain

I.J. Cabrillo, A. Calderon, B. Chazin Quero, J. Duarte Campderros, M. Fernandez, P.J. Fernández Manteca, A. García Alonso, G. Gomez, C. Martinez Rivero, P. Martinez Ruiz del Arbol, F. Matorras, J. Piedra Gomez, C. Prieels, F. Ricci-Tam, T. Rodrigo, A. Ruiz-Jimeno, L. Russo ${ }^{46}$, L. Scodellaro, I. Vila, J.M. Vizan Garcia

University of Colombo, Colombo, Sri Lanka

D.U.J. Sonnadara

University of Ruhuna, Department of Physics, Matara, Sri Lanka

W.G.D. Dharmaratna, N. Wickramage 
CERN, European Organization for Nuclear Research, Geneva, Switzerland T.K. Aarrestad, D. Abbaneo, B. Akgun, E. Auffray, G. Auzinger, J. Baechler, P. Baillon, A.H. Ball, D. Barney, J. Bendavid, M. Bianco, A. Bocci, P. Bortignon, E. Bossini, E. Brondolin, T. Camporesi, A. Caratelli, G. Cerminara, E. Chapon, G. Cucciati, D. d'Enterria, A. Dabrowski, N. Daci, V. Daponte, A. David, O. Davignon, A. De Roeck, M. Deile, R. Di Maria, M. Dobson, M. Dünser, N. Dupont, A. Elliott-Peisert, N. Emriskova, F. Fallavollita ${ }^{47}$, D. Fasanella, S. Fiorendi, G. Franzoni, J. Fulcher, W. Funk, S. Giani, D. Gigi, K. Gill, F. Glege, L. Gouskos, M. Gruchala, M. Guilbaud, D. Gulhan, J. Hegeman, C. Heidegger, Y. Iiyama, V. Innocente, T. James, P. Janot, O. Karacheban ${ }^{20}$, J. Kaspar, J. Kieseler, M. Krammer ${ }^{1}$, N. Kratochwil, C. Lange, P. Lecoq, K. Long, C. Lourenço, L. Malgeri, M. Mannelli, A. Massironi, F. Meijers, S. Mersi, E. Meschi, F. Moortgat, M. Mulders, J. Ngadiuba, J. Niedziela, S. Nourbakhsh, S. Orfanelli, L. Orsini, F. Pantaleo ${ }^{17}$, L. Pape, E. Perez, M. Peruzzi, A. Petrilli, G. Petrucciani, A. Pfeiffer, M. Pierini, F.M. Pitters, D. Rabady, A. Racz, M. Rieger, M. Rovere, H. Sakulin, J. Salfeld-Nebgen, S. Scarfi, C. Schäfer, C. Schwick, M. Selvaggi, A. Sharma, P. Silva, W. Snoeys, P. Sphicas ${ }^{48}$, J. Steggemann, S. Summers, V.R. Tavolaro, D. Treille, A. Tsirou, G.P. Van Onsem, A. Vartak, M. Verzetti, K.A. Wozniak, W.D. Zeuner

Paul Scherrer Institut, Villigen, Switzerland

L. Caminada ${ }^{49}$, K. Deiters, W. Erdmann, R. Horisberger, Q. Ingram, H.C. Kaestli, D. Kotlinski, U. Langenegger, T. Rohe

ETH Zurich - Institute for Particle Physics and Astrophysics (IPA), Zurich, Switzerland

M. Backhaus, P. Berger, A. Calandri, N. Chernyavskaya, G. Dissertori, M. Dittmar, M. Donegà, C. Dorfer, T.A. Gómez Espinosa, C. Grab, D. Hits, W. Lustermann, R.A. Manzoni, M.T. Meinhard, F. Micheli, P. Musella, F. Nessi-Tedaldi, F. Pauss, V. Perovic, G. Perrin, L. Perrozzi, S. Pigazzini, M.G. Ratti, M. Reichmann, C. Reissel, T. Reitenspiess, B. Ristic, D. Ruini, D.A. Sanz Becerra, M. Schönenberger, L. Shchutska, M.L. Vesterbacka Olsson, R. Wallny, D.H. Zhu

Universität Zürich, Zurich, Switzerland

C. Amsler ${ }^{50}$, C. Botta, D. Brzhechko, M.F. Canelli, A. De Cosa, R. Del Burgo, B. Kilminster, S. Leontsinis, V.M. Mikuni, I. Neutelings, G. Rauco, P. Robmann, K. Schweiger, Y. Takahashi, S. Wertz

National Central University, Chung-Li, Taiwan

C.M. Kuo, W. Lin, A. Roy, T. Sarkar ${ }^{29}$, S.S. Yu

National Taiwan University (NTU), Taipei, Taiwan

P. Chang, Y. Chao, K.F. Chen, P.H. Chen, W.-S. Hou, Y.y. Li, R.-S. Lu, E. Paganis, A. Psallidas, A. Steen

Chulalongkorn University, Faculty of Science, Department of Physics, Bangkok, Thailand

B. Asavapibhop, C. Asawatangtrakuldee, N. Srimanobhas, N. Suwonjandee 
Çukurova University, Physics Department, Science and Art Faculty, Adana, Turkey

A. Bat, F. Boran, A. Celik ${ }^{51}$, S. Damarseckin ${ }^{52}$, Z.S. Demiroglu, F. Dolek, C. Dozen ${ }^{53}$, I. Dumanoglu ${ }^{54}$, G. Gokbulut, Emine Gurpinar Guler ${ }^{55}$, Y. Guler, I. Hos ${ }^{56}$, C. Isik, E.E. Kangal ${ }^{57}$, O. Kara, A. Kayis Topaksu, U. Kiminsu, G. Onengut, K. Ozdemir ${ }^{58}$, A.E. Simsek, U.G. Tok, S. Turkcapar, I.S. Zorbakir, C. Zorbilmez

Middle East Technical University, Physics Department, Ankara, Turkey B. Isildak ${ }^{59}$, G. Karapinar ${ }^{60}$, M. Yalvac ${ }^{61}$

Bogazici University, Istanbul, Turkey

I.O. Atakisi, E. Gülmez, M. Kaya ${ }^{62}$, O. Kaya ${ }^{63}$, Ö. Özçelik, S. Tekten ${ }^{64}$, E.A. Yetkin ${ }^{65}$

Istanbul Technical University, Istanbul, Turkey

A. Cakir, K. Cankocak ${ }^{54}$, Y. Komurcu, S. Sen ${ }^{66}$

Istanbul University, Istanbul, Turkey

S. Cerci ${ }^{67}$, B. Kaynak, S. Ozkorucuklu, D. Sunar Cerci ${ }^{67}$

Institute for Scintillation Materials of National Academy of Science of Ukraine, Kharkov, Ukraine

B. Grynyov

National Scientific Center, Kharkov Institute of Physics and Technology, Kharkov, Ukraine

L. Levchuk

University of Bristol, Bristol, United Kingdom

E. Bhal, S. Bologna, J.J. Brooke, D. Burns ${ }^{68}$, E. Clement, D. Cussans, H. Flacher,

J. Goldstein, G.P. Heath, H.F. Heath, L. Kreczko, B. Krikler, S. Paramesvaran, T. Sakuma,

S. Seif El Nasr-Storey, V.J. Smith, J. Taylor, A. Titterton

Rutherford Appleton Laboratory, Didcot, United Kingdom

K.W. Bell, A. Belyaev ${ }^{69}$, C. Brew, R.M. Brown, D.J.A. Cockerill, J.A. Coughlan, K. Harder, S. Harper, J. Linacre, K. Manolopoulos, D.M. Newbold, E. Olaiya, D. Petyt, T. Reis, T. Schuh, C.H. Shepherd-Themistocleous, A. Thea, I.R. Tomalin, T. Williams

Imperial College, London, United Kingdom

R. Bainbridge, P. Bloch, S. Bonomally, J. Borg, S. Breeze, O. Buchmuller, A. Bundock, Gurpreet Singh CHAHAL ${ }^{70}$, D. Colling, P. Dauncey, G. Davies, M. Della Negra, P. Everaerts, G. Hall, G. Iles, M. Komm, J. Langford, L. Lyons, A.-M. Magnan, S. Malik, A. Martelli, V. Milosevic, A. Morton, J. Nash ${ }^{71}$, V. Palladino, M. Pesaresi, D.M. Raymond, A. Richards, A. Rose, E. Scott, C. Seez, A. Shtipliyski, M. Stoye, T. Strebler, A. Tapper, K. Uchida, T. Virdee ${ }^{17}$, N. Wardle, S.N. Webb, D. Winterbottom, A.G. Zecchinelli, S.C. Zenz

Brunel University, Uxbridge, United Kingdom

J.E. Cole, P.R. Hobson, A. Khan, P. Kyberd, C.K. Mackay, I.D. Reid, L. Teodorescu, S. Zahid 
Baylor University, Waco, U.S.A.

A. Brinkerhoff, K. Call, B. Caraway, J. Dittmann, K. Hatakeyama, C. Madrid, B. McMaster, N. Pastika, C. Smith

Catholic University of America, Washington, DC, U.S.A.

R. Bartek, A. Dominguez, R. Uniyal, A.M. Vargas Hernandez

The University of Alabama, Tuscaloosa, U.S.A.

A. Buccilli, S.I. Cooper, S.V. Gleyzer, C. Henderson, P. Rumerio, C. West

Boston University, Boston, U.S.A.

A. Albert, D. Arcaro, Z. Demiragli, D. Gastler, C. Richardson, J. Rohlf, D. Sperka, D. Spitzbart, I. Suarez, L. Sulak, D. Zou

Brown University, Providence, U.S.A.

G. Benelli, B. Burkle, X. Coubez ${ }^{18}$, D. Cutts, Y.t. Duh, M. Hadley, U. Heintz, J.M. Hogan ${ }^{72}$, K.H.M. Kwok, E. Laird, G. Landsberg, K.T. Lau, J. Lee, M. Narain, S. Sagir ${ }^{73}$, R. Syarif, E. Usai, W.Y. Wong, D. Yu, W. Zhang

University of California, Davis, Davis, U.S.A.

R. Band, C. Brainerd, R. Breedon, M. Calderon De La Barca Sanchez, M. Chertok, J. Conway, R. Conway, P.T. Cox, R. Erbacher, C. Flores, G. Funk, F. Jensen, W. Ko ${ }^{\dagger}$, O. Kukral, R. Lander, M. Mulhearn, D. Pellett, J. Pilot, M. Shi, D. Taylor, K. Tos, M. Tripathi, Z. Wang, F. Zhang

University of California, Los Angeles, U.S.A.

M. Bachtis, C. Bravo, R. Cousins, A. Dasgupta, A. Florent, J. Hauser, M. Ignatenko, N. Mccoll, W.A. Nash, S. Regnard, D. Saltzberg, C. Schnaible, B. Stone, V. Valuev

University of California, Riverside, Riverside, U.S.A.

K. Burt, Y. Chen, R. Clare, J.W. Gary, S.M.A. Ghiasi Shirazi, G. Hanson, G. Karapostoli, O.R. Long, N. Manganelli, M. Olmedo Negrete, M.I. Paneva, W. Si, S. Wimpenny, B.R. Yates, Y. Zhang

University of California, San Diego, La Jolla, U.S.A.

J.G. Branson, P. Chang, S. Cittolin, S. Cooperstein, N. Deelen, M. Derdzinski, J. Duarte, R. Gerosa, D. Gilbert, B. Hashemi, D. Klein, V. Krutelyov, J. Letts, M. Masciovecchio, S. May, S. Padhi, M. Pieri, V. Sharma, M. Tadel, F. Würthwein, A. Yagil, G. Zevi Della Porta

University of California, Santa Barbara - Department of Physics, Santa Barbara, U.S.A.

N. Amin, R. Bhandari, C. Campagnari, M. Citron, V. Dutta, J. Incandela, B. Marsh, H. Mei, A. Ovcharova, H. Qu, J. Richman, U. Sarica, D. Stuart, S. Wang

California Institute of Technology, Pasadena, U.S.A.

D. Anderson, A. Bornheim, O. Cerri, I. Dutta, J.M. Lawhorn, N. Lu, J. Mao, H.B. Newman, T.Q. Nguyen, J. Pata, M. Spiropulu, J.R. Vlimant, S. Xie, Z. Zhang, R.Y. Zhu 
Carnegie Mellon University, Pittsburgh, U.S.A.

J. Alison, M.B. Andrews, T. Ferguson, T. Mudholkar, M. Paulini, M. Sun, I. Vorobiev, M. Weinberg

University of Colorado Boulder, Boulder, U.S.A.

J.P. Cumalat, W.T. Ford, E. MacDonald, T. Mulholland, R. Patel, A. Perloff, K. Stenson, K.A. Ulmer, S.R. Wagner

Cornell University, Ithaca, U.S.A.

J. Alexander, Y. Cheng, J. Chu, A. Datta, A. Frankenthal, K. Mcdermott, J.R. Patterson,

D. Quach, A. Ryd, S.M. Tan, Z. Tao, J. Thom, P. Wittich, M. Zientek

Fermi National Accelerator Laboratory, Batavia, U.S.A.

S. Abdullin, M. Albrow, M. Alyari, G. Apollinari, A. Apresyan, A. Apyan, S. Banerjee, L.A.T. Bauerdick, A. Beretvas, D. Berry, J. Berryhill, P.C. Bhat, K. Burkett, J.N. Butler, A. Canepa, G.B. Cerati, H.W.K. Cheung, F. Chlebana, M. Cremonesi, V.D. Elvira, J. Freeman, Z. Gecse, E. Gottschalk, L. Gray, D. Green, S. Grünendahl, O. Gutsche, J. Hanlon, R.M. Harris, S. Hasegawa, R. Heller, J. Hirschauer, B. Jayatilaka, S. Jindariani, M. Johnson, U. Joshi, T. Klijnsma, B. Klima, M.J. Kortelainen, B. Kreis, S. Lammel, J. Lewis, D. Lincoln, R. Lipton, M. Liu, T. Liu, J. Lykken, K. Maeshima, J.M. Marraffino, D. Mason, P. McBride, P. Merkel, S. Mrenna, S. Nahn, V. O’Dell, V. Papadimitriou, K. Pedro, C. Pena ${ }^{74}$, F. Ravera, A. Reinsvold Hall, L. Ristori, B. Schneider, E. SextonKennedy, N. Smith, A. Soha, W.J. Spalding, L. Spiegel, S. Stoynev, J. Strait, L. Taylor, S. Tkaczyk, N.V. Tran, L. Uplegger, E.W. Vaandering, R. Vidal, M. Wang, H.A. Weber, A. Woodard

University of Florida, Gainesville, U.S.A.

D. Acosta, P. Avery, D. Bourilkov, L. Cadamuro, V. Cherepanov, F. Errico, R.D. Field, D. Guerrero, B.M. Joshi, M. Kim, J. Konigsberg, A. Korytov, K.H. Lo, K. Matchev, N. Menendez, G. Mitselmakher, D. Rosenzweig, K. Shi, J. Wang, S. Wang, X. Zuo

Florida International University, Miami, U.S.A.

Y.R. Joshi

Florida State University, Tallahassee, U.S.A.

T. Adams, A. Askew, S. Hagopian, V. Hagopian, K.F. Johnson, R. Khurana, T. Kolberg, G. Martinez, T. Perry, H. Prosper, C. Schiber, R. Yohay, J. Zhang

Florida Institute of Technology, Melbourne, U.S.A.

M.M. Baarmand, M. Hohlmann, D. Noonan, M. Rahmani, M. Saunders, F. Yumiceva

University of Illinois at Chicago (UIC), Chicago, U.S.A.

M.R. Adams, L. Apanasevich, R.R. Betts, R. Cavanaugh, X. Chen, S. Dittmer, O. Evdokimov, C.E. Gerber, D.A. Hangal, D.J. Hofman, V. Kumar, C. Mills, G. Oh, T. Roy, M.B. Tonjes, N. Varelas, J. Viinikainen, H. Wang, X. Wang, Z. Wu 
The University of Iowa, Iowa City, U.S.A.

M. Alhusseini, B. Bilki ${ }^{55}$, K. Dilsiz ${ }^{75}$, S. Durgut, R.P. Gandrajula, M. Haytmyradov, V. Khristenko, O.K. Köseyan, J.-P. Merlo, A. Mestvirishvili ${ }^{76}$, A. Moeller, J. Nachtman, H. Ogul ${ }^{77}$, Y. Onel, F. Ozok ${ }^{78}$, A. Penzo, C. Snyder, E. Tiras, J. Wetzel, K. Yi ${ }^{79}$

Johns Hopkins University, Baltimore, U.S.A.

B. Blumenfeld, A. Cocoros, N. Eminizer, A.V. Gritsan, W.T. Hung, S. Kyriacou, P. Maksimovic, C. Mantilla, J. Roskes, M. Swartz, T.Á. Vámi

The University of Kansas, Lawrence, U.S.A.

C. Baldenegro Barrera, P. Baringer, A. Bean, S. Boren, A. Bylinkin, T. Isidori, S. Khalil,

J. King, G. Krintiras, A. Kropivnitskaya, C. Lindsey, D. Majumder, W. Mcbrayer,

N. Minafra, M. Murray, C. Rogan, C. Royon, S. Sanders, E. Schmitz, J.D. Tapia Takaki,

Q. Wang, J. Williams, G. Wilson

Kansas State University, Manhattan, U.S.A.

S. Duric, A. Ivanov, K. Kaadze, D. Kim, Y. Maravin, D.R. Mendis, T. Mitchell, A. Modak, A. Mohammadi

Lawrence Livermore National Laboratory, Livermore, U.S.A.

F. Rebassoo, D. Wright

University of Maryland, College Park, U.S.A.

A. Baden, O. Baron, A. Belloni, S.C. Eno, Y. Feng, N.J. Hadley, S. Jabeen, G.Y. Jeng, R.G. Kellogg, A.C. Mignerey, S. Nabili, M. Seidel, A. Skuja, S.C. Tonwar, L. Wang, K. Wong

Massachusetts Institute of Technology, Cambridge, U.S.A.

D. Abercrombie, B. Allen, R. Bi, S. Brandt, W. Busza, I.A. Cali, Y. Chen, M. D'Alfonso,

G. Gomez Ceballos, M. Goncharov, P. Harris, D. Hsu, M. Hu, M. Klute, D. Kovalskyi, Y.-J. Lee, P.D. Luckey, B. Maier, A.C. Marini, C. Mcginn, C. Mironov, S. Narayanan, X. Niu, C. Paus, D. Rankin, C. Roland, G. Roland, Z. Shi, G.S.F. Stephans, K. Sumorok, K. Tatar, M. Taylor, D. Velicanu, J. Wang, T.W. Wang, B. Wyslouch

University of Minnesota, Minneapolis, U.S.A.

R.M. Chatterjee, A. Evans, S. Guts ${ }^{\dagger}$, P. Hansen, J. Hiltbrand, Sh. Jain, Y. Kubota, Z. Lesko, J. Mans, M. Revering, R. Rusack, R. Saradhy, N. Schroeder, N. Strobbe, M.A. Wadud

University of Mississippi, Oxford, U.S.A.

J.G. Acosta, S. Oliveros

University of Nebraska-Lincoln, Lincoln, U.S.A.

K. Bloom, S. Chauhan, D.R. Claes, C. Fangmeier, L. Finco, F. Golf, R. Kamalieddin, I. Kravchenko, J.E. Siado, G.R. Snow ${ }^{\dagger}$, B. Stieger, W. Tabb

State University of New York at Buffalo, Buffalo, U.S.A.

G. Agarwal, C. Harrington, I. Iashvili, A. Kharchilava, C. McLean, D. Nguyen, A. Parker, J. Pekkanen, S. Rappoccio, B. Roozbahani 
Northeastern University, Boston, U.S.A.

G. Alverson, E. Barberis, C. Freer, Y. Haddad, A. Hortiangtham, G. Madigan, B. Marzocchi, D.M. Morse, V. Nguyen, T. Orimoto, L. Skinnari, A. Tishelman-Charny, T. Wamorkar, B. Wang, A. Wisecarver, D. Wood

Northwestern University, Evanston, U.S.A.

S. Bhattacharya, J. Bueghly, G. Fedi, A. Gilbert, T. Gunter, K.A. Hahn, N. Odell, M.H. Schmitt, K. Sung, M. Velasco

University of Notre Dame, Notre Dame, U.S.A.

R. Bucci, N. Dev, R. Goldouzian, M. Hildreth, K. Hurtado Anampa, C. Jessop, D.J. Karmgard, K. Lannon, W. Li, N. Loukas, N. Marinelli, I. Mcalister, F. Meng, Y. Musienko ${ }^{37}$, R. Ruchti, P. Siddireddy, G. Smith, S. Taroni, M. Wayne, A. Wightman, M. Wolf

The Ohio State University, Columbus, U.S.A.

J. Alimena, B. Bylsma, B. Cardwell, L.S. Durkin, B. Francis, C. Hill, W. Ji, A. Lefeld, T.Y. Ling, B.L. Winer

Princeton University, Princeton, U.S.A.

G. Dezoort, P. Elmer, J. Hardenbrook, N. Haubrich, S. Higginbotham, A. Kalogeropoulos,

S. Kwan, D. Lange, M.T. Lucchini, J. Luo, D. Marlow, K. Mei, I. Ojalvo, J. Olsen, C. Palmer, P. Piroué, D. Stickland, C. Tully

University of Puerto Rico, Mayaguez, U.S.A.

S. Malik, S. Norberg

Purdue University, West Lafayette, U.S.A.

A. Barker, V.E. Barnes, R. Chawla, S. Das, L. Gutay, M. Jones, A.W. Jung, B. Mahakud, D.H. Miller, G. Negro, N. Neumeister, C.C. Peng, S. Piperov, H. Qiu, J.F. Schulte, N. Trevisani, F. Wang, R. Xiao, W. Xie

Purdue University Northwest, Hammond, U.S.A.

T. Cheng, J. Dolen, N. Parashar

Rice University, Houston, U.S.A.

A. Baty, U. Behrens, S. Dildick, K.M. Ecklund, S. Freed, F.J.M. Geurts, M. Kilpatrick, Arun Kumar, W. Li, B.P. Padley, R. Redjimi, J. Roberts, J. Rorie, W. Shi, A.G. Stahl Leiton, Z. Tu, A. Zhang

University of Rochester, Rochester, U.S.A.

A. Bodek, P. de Barbaro, R. Demina, J.L. Dulemba, C. Fallon, T. Ferbel, M. Galanti,

A. Garcia-Bellido, O. Hindrichs, A. Khukhunaishvili, E. Ranken, R. Taus

Rutgers, The State University of New Jersey, Piscataway, U.S.A.

B. Chiarito, J.P. Chou, A. Gandrakota, Y. Gershtein, E. Halkiadakis, A. Hart, M. Heindl,

E. Hughes, S. Kaplan, I. Laflotte, A. Lath, R. Montalvo, K. Nash, M. Osherson, S. Salur,

S. Schnetzer, S. Somalwar, R. Stone, S. Thomas 
University of Tennessee, Knoxville, U.S.A.

H. Acharya, A.G. Delannoy, S. Spanier

Texas A\&M University, College Station, U.S.A.

O. Bouhali ${ }^{80}$, M. Dalchenko, M. De Mattia, A. Delgado, R. Eusebi, J. Gilmore, T. Huang,

T. Kamon ${ }^{81}$, H. Kim, S. Luo, S. Malhotra, D. Marley, R. Mueller, D. Overton, L. Perniè,

D. Rathjens, A. Safonov

Texas Tech University, Lubbock, U.S.A.

N. Akchurin, J. Damgov, F. De Guio, V. Hegde, S. Kunori, K. Lamichhane, S.W. Lee, T. Mengke, S. Muthumuni, T. Peltola, S. Undleeb, I. Volobouev, Z. Wang, A. Whitbeck

Vanderbilt University, Nashville, U.S.A.

S. Greene, A. Gurrola, R. Janjam, W. Johns, C. Maguire, A. Melo, H. Ni, K. Padeken, F. Romeo, P. Sheldon, S. Tuo, J. Velkovska, M. Verweij

University of Virginia, Charlottesville, U.S.A.

M.W. Arenton, P. Barria, B. Cox, G. Cummings, J. Hakala, R. Hirosky, M. Joyce, A. Ledovskoy, C. Neu, B. Tannenwald, Y. Wang, E. Wolfe, F. Xia

Wayne State University, Detroit, U.S.A.

R. Harr, P.E. Karchin, N. Poudyal, J. Sturdy, P. Thapa

University of Wisconsin - Madison, Madison, WI, U.S.A.

K. Black, T. Bose, J. Buchanan, C. Caillol, D. Carlsmith, S. Dasu, I. De Bruyn, L. Dodd, C. Galloni, H. He, M. Herndon, A. Hervé, U. Hussain, A. Lanaro, A. Loeliger, R. Loveless, J. Madhusudanan Sreekala, A. Mallampalli, D. Pinna, T. Ruggles, A. Savin, V. Sharma, W.H. Smith, D. Teague, S. Trembath-Reichert

$\dagger$ : Deceased

1: Also at Vienna University of Technology, Vienna, Austria

2: Also at Université Libre de Bruxelles, Bruxelles, Belgium

3: Also at IRFU, CEA, Université Paris-Saclay, Gif-sur-Yvette, France

4: Also at Universidade Estadual de Campinas, Campinas, Brazil

5: Also at Federal University of Rio Grande do Sul, Porto Alegre, Brazil

6: Also at UFMS, Nova Andradina, Brazil

7: Also at Universidade Federal de Pelotas, Pelotas, Brazil

8: Also at University of Chinese Academy of Sciences, Beijing, China

9: Also at Institute for Theoretical and Experimental Physics named by A.I. Alikhanov of NRC 'Kurchatov Institute', Moscow, Russia

10: Also at Joint Institute for Nuclear Research, Dubna, Russia

11: Also at Fayoum University, El-Fayoum, Egypt

12: Now at British University in Egypt, Cairo, Egypt

13: Also at Purdue University, West Lafayette, U.S.A.

14: Also at Université de Haute Alsace, Mulhouse, France

15: Also at Ilia State University, Tbilisi, Georgia

16: Also at Erzincan Binali Yildirim University, Erzincan, Turkey

17: Also at CERN, European Organization for Nuclear Research, Geneva, Switzerland 
18: Also at RWTH Aachen University, III. Physikalisches Institut A, Aachen, Germany

19: Also at University of Hamburg, Hamburg, Germany

20: Also at Brandenburg University of Technology, Cottbus, Germany

21: Also at Institute of Physics, University of Debrecen, Debrecen, Hungary, Debrecen, Hungary

22: Also at Institute of Nuclear Research ATOMKI, Debrecen, Hungary

23: Also at MTA-ELTE Lendület CMS Particle and Nuclear Physics Group, Eötvös Loránd University, Budapest, Hungary, Budapest, Hungary

24: Also at IIT Bhubaneswar, Bhubaneswar, India, Bhubaneswar, India

25: Also at Institute of Physics, Bhubaneswar, India

26: Also at G.H.G. Khalsa College, Punjab, India

27: Also at Shoolini University, Solan, India

28: Also at University of Hyderabad, Hyderabad, India

29: Also at University of Visva-Bharati, Santiniketan, India

30: Now at INFN Sezione di Bari ${ }^{a}$, Università di Bari ${ }^{b}$, Politecnico di Bari ${ }^{c}$, Bari, Italy

31: Also at Italian National Agency for New Technologies, Energy and Sustainable Economic Development, Bologna, Italy

32: Also at Centro Siciliano di Fisica Nucleare e di Struttura Della Materia, Catania, Italy

33: Also at Riga Technical University, Riga, Latvia, Riga, Latvia

34: Also at Malaysian Nuclear Agency, MOSTI, Kajang, Malaysia

35: Also at Consejo Nacional de Ciencia y Tecnología, Mexico City, Mexico

36: Also at Warsaw University of Technology, Institute of Electronic Systems, Warsaw, Poland

37: Also at Institute for Nuclear Research, Moscow, Russia

38: Now at National Research Nuclear University 'Moscow Engineering Physics Institute' (MEPhI), Moscow, Russia

39: Also at St. Petersburg State Polytechnical University, St. Petersburg, Russia

40: Also at University of Florida, Gainesville, U.S.A.

41: Also at Imperial College, London, United Kingdom

42: Also at P.N. Lebedev Physical Institute, Moscow, Russia

43: Also at INFN Sezione di Padova ${ }^{a}$, Università di Padova ${ }^{b}$, Padova, Italy, Università di Trento ${ }^{c}$, Trento, Italy, Padova, Italy

44: Also at Budker Institute of Nuclear Physics, Novosibirsk, Russia

45: Also at Faculty of Physics, University of Belgrade, Belgrade, Serbia

46: Also at Università degli Studi di Siena, Siena, Italy, Siena, Italy

47: Also at INFN Sezione di Pavia ${ }^{a}$, Università di Pavia ${ }^{b}$, Pavia, Italy, Pavia, Italy

48: Also at National and Kapodistrian University of Athens, Athens, Greece

49: Also at Universität Zürich, Zurich, Switzerland

50: Also at Stefan Meyer Institute for Subatomic Physics, Vienna, Austria, Vienna, Austria

51: Also at Burdur Mehmet Akif Ersoy University, BURDUR, Turkey

52: Also at Şırnak University, Sirnak, Turkey

53: Also at Department of Physics, Tsinghua University, Beijing, China, Beijing, China

54: Also at Near East University, Research Center of Experimental Health Science, Nicosia, Turkey

55: Also at Beykent University, Istanbul, Turkey, Istanbul, Turkey

56: Also at Istanbul Aydin University, Application and Research Center for Advanced Studies (App. \& Res. Cent. for Advanced Studies), Istanbul, Turkey

57: Also at Mersin University, Mersin, Turkey

58: Also at Piri Reis University, Istanbul, Turkey

59: Also at Ozyegin University, Istanbul, Turkey 
60: Also at Izmir Institute of Technology, Izmir, Turkey

61: Also at Bozok Universitetesi Rektörlügü, Yozgat, Turkey, Yozgat, Turkey

62: Also at Marmara University, Istanbul, Turkey

63: Also at Milli Savunma University, Istanbul, Turkey

64: Also at Kafkas University, Kars, Turkey

65: Also at Istanbul Bilgi University, Istanbul, Turkey

66: Also at Hacettepe University, Ankara, Turkey

67: Also at Adiyaman University, Adiyaman, Turkey

68: Also at Vrije Universiteit Brussel, Brussel, Belgium

69: Also at School of Physics and Astronomy, University of Southampton, Southampton, United Kingdom

70: Also at IPPP Durham University, Durham, United Kingdom

71: Also at Monash University, Faculty of Science, Clayton, Australia

72: Also at Bethel University, St. Paul, Minneapolis, U.S.A.

73: Also at Karamanoğlu Mehmetbey University, Karaman, Turkey

74: Also at California Institute of Technology, Pasadena, U.S.A.

75: Also at Bingol University, Bingol, Turkey

76: Also at Georgian Technical University, Tbilisi, Georgia

77: Also at Sinop University, Sinop, Turkey

78: Also at Mimar Sinan University, Istanbul, Istanbul, Turkey

79: Also at Nanjing Normal University Department of Physics, Nanjing, China

80: Also at Texas A\&M University at Qatar, Doha, Qatar

81: Also at Kyungpook National University, Daegu, Korea, Daegu, Korea 